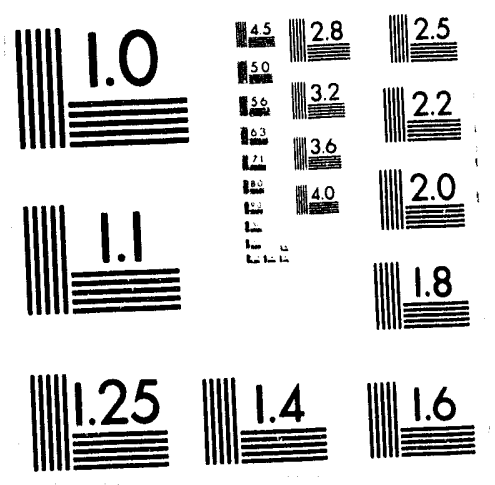



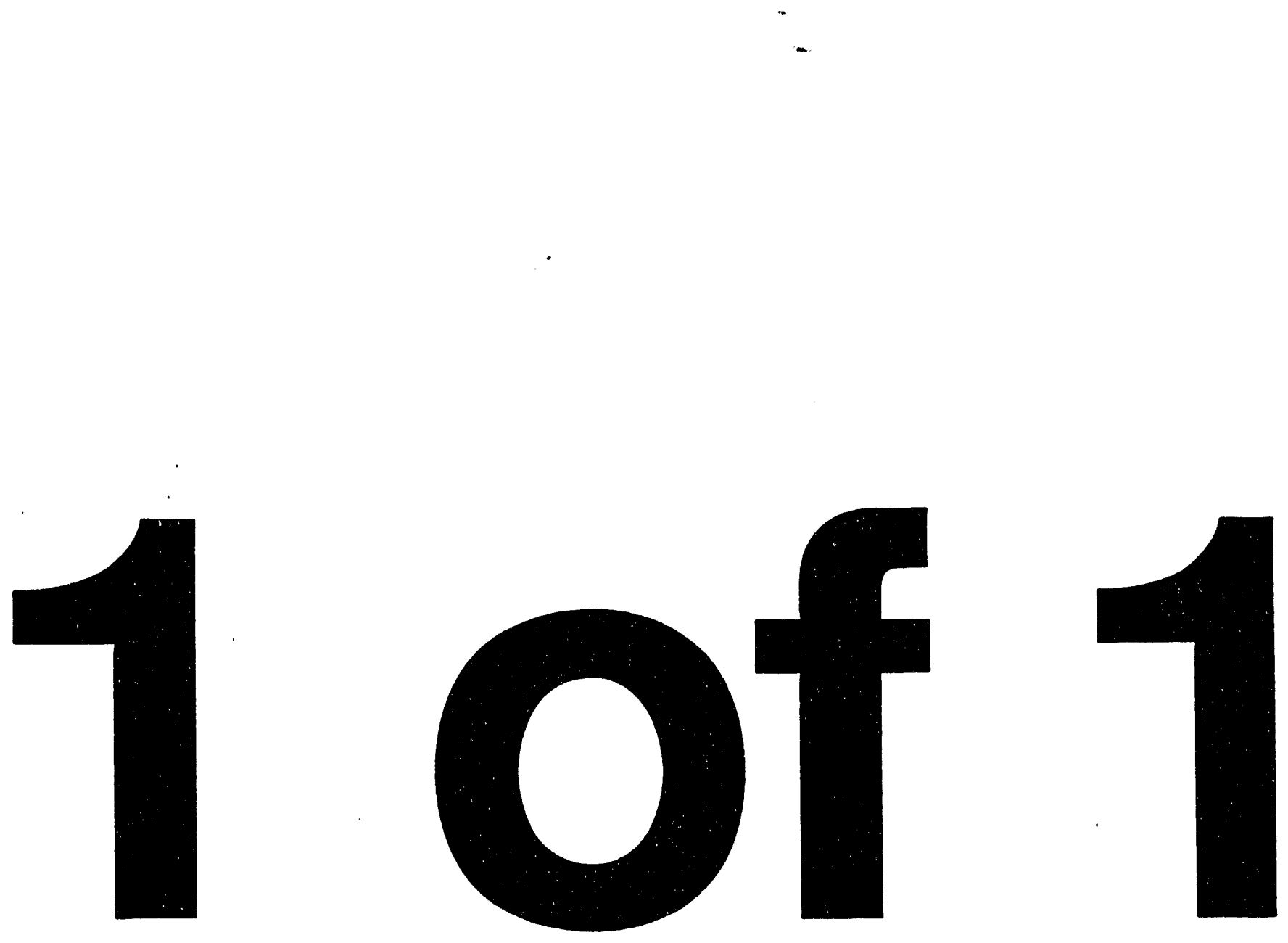


\section{SANDIA REPORT}

SAND93-2765 • UC-902

Unlimited Release

Printed March 1994

\section{The In Situ Permeable Flow Sensor: A Device for Measuring Groundwater Flow Velocity}

Sanford Ballard, Glenn T. Barker, Ralph L. Nichols

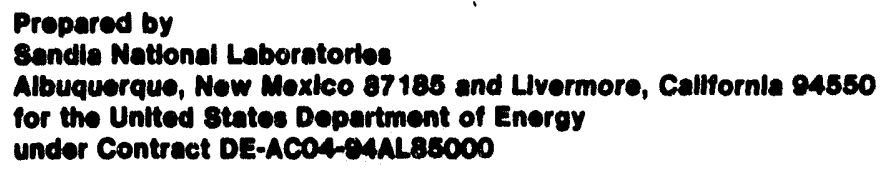

Approved for public release; diatribution lo unlimited. 
Iseued by Sandia National Laboratories, operated for the United States Department of Energy by Sandia Corporation.

NOTICE: This report was prepared as an account of work sponsored ky an agency of the United States Government. Neither the United States Government nor any agency thereof, nor any of their employees, nor any of their contractors, subcontractors, or their employees, makes any warranty, express or implied, or assumes any legal liability or responsibility for the accuracy, completeness, or usefulness of any information, apparatus, product, or process disclosed, or represents that its use would not infringe privately owned rights. Reference herein to any specific commercial product, process, or service by trade name, trademark, manufacturer, or otherwise, does not necessarily constitute or imply its endorsement, recommendation, or favoring by the United States Government, any agency thereof or any of their contractors or subcontractors. The views and opinions expressed herein do not necessarily state or reflect those of the United States Government, any agency thereof or any of their contractors.

Printed in the United States of America. This report has been reproduced directly from the best available copy.

Available to DOE and DOE contractors from

Office of Scientific and Technical Information

PO Box 62

Oak Ridge, TN 37831

Prices available from (615) 576-8401, FTS 626-8401

Available to the public from

National Technical Information Service

US Department of Commerce

5285 Port Royal Rd

Springfield, VA 22161

NTIS price codes

Printed copy: A03

Microfiche copy: A01 
SAND93-2765

Distribution

Unlimited Release

Printed March, 1994

Category UC-902

\title{
The In Situ Permeable flow Sensor: A Device for Measuring Groundwater Flow Velocity
}

\author{
Sanford Ballard and Glenn T. Barker \\ Geophysics Department \\ Sandia National Laboratories \\ Albuquerque, NM 87185-0750 \\ Ralph L. Nichols \\ Environmental Sciences Section \\ Savannah River Technology Center \\ Aiken, SC 29808
}

\begin{abstract}
A new technology called the In Situ Permeable Flow Sensor has been developed at Sandia National Laboratories. These sensors use a thermal perturbation technique to directly measure the direction and magnitude of the full three dimensional groundwater flow velocity vector in unconsolidated, saturated, porous media. The velocity measured is an average value characteristic of an approximately 1 cubic meter volume of the subsurface. During a test at the Savannah River Site in South Carolina, two flow sensors were deployed in a confined aquifer in close proximity to a well which was screened over the entire vertical extent of the aquifer and the well was pumped at four different pumping rates. In this situation horizontal flow which is radially directed toward the pumping well is expected. The flow sensors measured horizontal flow which was directed toward the pumping well, within the uncertainty in the measurements. The observed magnitude of the horizontal component of the flow velocity increased linearly with pumping rate, as predicted by theoretical considerations. The measured horizontal component of the flow velocity differed from the predicted flow velocity, which was calculated with the assumptions that the hydraulic properties of the aquifer were radially homogeneous and isotropic, by less than a factor of two. Drawdown data obtained from other wells near the pumping well during the pump test indicate that the hydraulic properties of the aquifer are probably not radially homogeneous but the effect of the inhomogeneity on the flow velocity field around the pumping well was not modeled because the degree and distribution of the inhomogeneity are unknown. Grain size analysis of core samples from wells in the area were used to estimate the vertical distribution of hydraulic conductivity. Vertical components of groundwater flow observed with the flow sensors are qualitatively consistent with the vertical distribution of horizontal hydraulic conductivity estimated from grain size analysis but are significantly larger in magnitude than predicted. This is likely due to the creation of a vertical conduit of increased hydraulic conductivity during emplacement of the probes. Overall, the flow sensors performed very well.
\end{abstract}

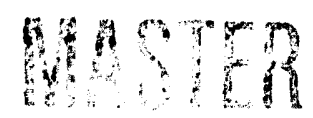




\section{Acknowledgements}

The authors thank Louis Romero for many helpful discussions related to the mathematical aspects of flow sensor data interpretation and Marianne Walck, Mike Nichols and Eric Webb for reviewing the manuscript. This work was funded by the Department of Energy Office of Technology Development under Technical Task Plan number AL-2011-01. 


\section{Contents}

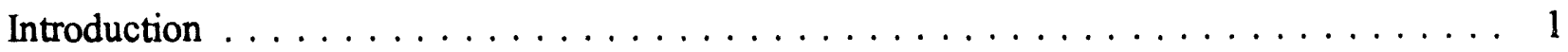

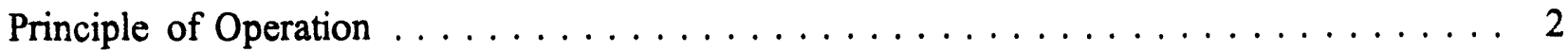

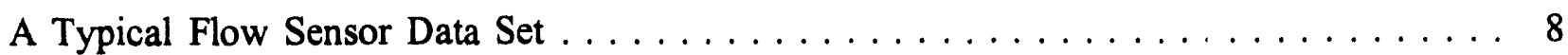

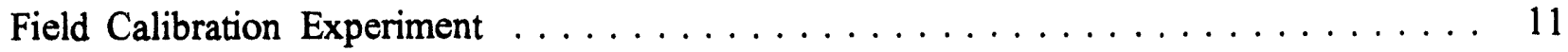

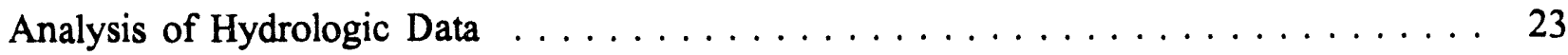

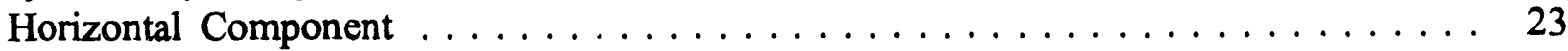

Vertical Component . . . . . . . . . . . . . . . . . . . . 27

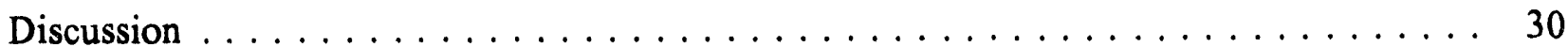

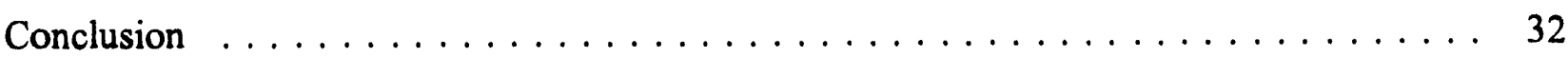

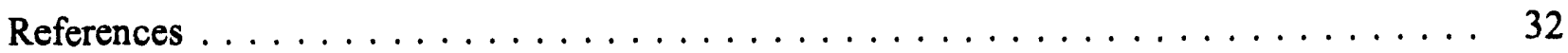

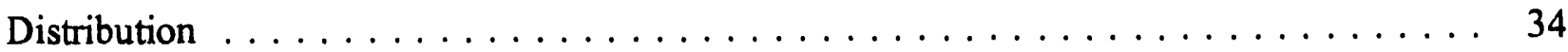




\section{List of Figures}

Figure 1 - a) Temperature as a function of azimuth for a probe in a purely horizontal flow field.

Figure 2 - Contour maps of theoretical probe surface temperatures.

Figure 3 - An In Situ Permeable Flow Sensor being emplaced into the subsurface through

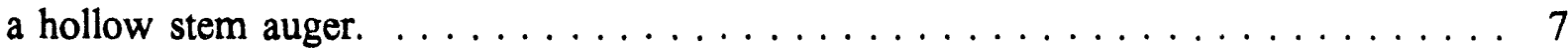

Figure 4 - Distribution pattern of the 30 thermistor locations on the surface of the probes. 7

Figure 5 - a) Temperature on the surface of the probe, b) deviation from the average temperature and c) corrected deviation from the average temperature as a function of time.

Figure 6 - Illustration of the technique used to determine correction factors. . . . . 10

Figure 7 - Temperature as a function of a) azimuth and b) vertical position on the probe surface.

Figure 8 - a) Map and b) cross section of the field test site at Savannah River. . . . . .

Figure 9 - a) Temperature and b) corrected deviation fiom the average temperature on the surface of the probe in TMF2B $(R=15.5 \mathrm{ft})$ at 6 and $3 \mathrm{gpm}$. c) Temperature as a function of azimuth for the data from ring 3 .

Figure 10 - Flow velocity measured by the probe in TMF2B $(R=15.5 \mathrm{ft})$ when the pumping rate was 6 and then $3 \mathrm{gpm} . \ldots \ldots \ldots \ldots \ldots \ldots$

Figure 11 - Flow velocity measured by the probe in TMF2B $(R=15.5 \mathrm{ft}$ ) when the pumping rate was 15 and then $9 \mathrm{gpm}$.

Figure 12 - Flow velocity measured by the probe in TMF1B $(R=38 \mathrm{ft})$ when the pumping

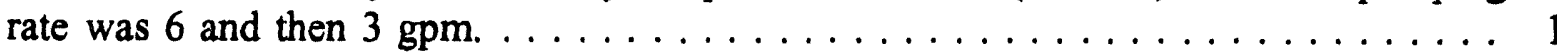

Figure 13 - Flow velocity measured by the probe in TMF1B $(R=38 \mathrm{ft})$ when the pumping rate was 15.5 and then $9 \mathrm{gpm} . \ldots \ldots \ldots \ldots \ldots \ldots \ldots \ldots$

Figure 14 - Flow velocity as a function of pumping rate for the two probes. . . . . 20

Figure 15 - Approximate time required for measured horizontal magnitude to reach

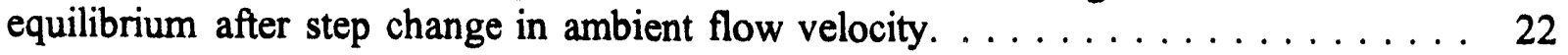

Figure 16 - Cylinders of constant flow velocity surrounding the pumping well. . . . . 23 
Figure 17 - Comparison of measured and theoretical horizontal flow velocities. . . . . . 24

Figure 18 - Drawdown vs. log time at different distances from the pumping well $\ldots \ldots 26$

Figure 19 - Drawdown vs radial distance from the pumping well for several different

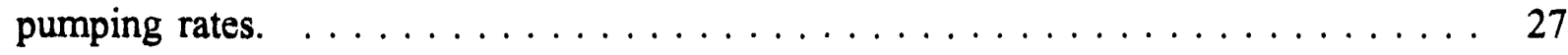

Figure 20 - Comparison of observed and modeled hydraulic gradients $\ldots \ldots \ldots 27$

Figure 21 - Vertical hydraulic conductivity distribution in the aquifer $\ldots \ldots \ldots$

Figure 22 - The vertical component of the flow velocity and the downward deviation from the horizontal vs depth . . . . . . . . . . . . . . . . . . . . . . . 29

\section{List of Tables}

Table 1 - Flow velocity measured at TMF1B 38 feet from the pumping well. . . . . . 21

Table 2 - Flow velocity measured at TMF2B 15.5 feet from the pumping well. . . . . 21

Table 3 - Hydrologic properties of the relevant formations at the test site. . . . . . . 24 
Intentionally Left Blank 


\section{Introduction}

A new instrument which uses a thermal perturbation technique to directly measure the three dimensional groundwater flow velocity vector in unconsolidated, saturated, porous media has been developed at Sandia National Laboratories (Ballard, 1992a, 1992b). Since groundwater flow is perhaps the most important mechanism for the dispersal of many types of toxic waste once they have been released into the subsurface, accurate information about the groundwater flow field is critical to the characterization of waste sites, monitoring of waste remediation activities and monitoring the post-closure performance of remediated waste sites. In addition, the technology will be very useful in hydrologic studies investigating scales and distribution of aquifer heterogeneity.

This technology enjoys a number of advantages over the currently accepted method of obtaining flow velocity information which involves making hydraulic head measurements in a number of screened boreholes and from that data determining the hydraulic gradients in the subsurface. With hydraulic conductivity data, the velocity field between the boreholes can be modeled. While this approach is entirely appropriate in many circumstances, it suffers from several disadvantages in certain instances. The first disadvantage of the standard practice is that detailed knowledge of the hydraulic conductivity distribution in the subsurface is required. Hydraulic conductivity is generally determined with either a pump test or a slug test. In a pump test water is pumped from a well while in a slug test, water is injected into a well. In both cases the hydraulic conductivity of the sediments in the range of influence of the test can be determined from the time rate of change of water levels in nearby holes. In areas where the groundwater is contaminated, conducting these types of test can be very undesirable. In the case of a pump test the water must be disposed of as hazardous waste, which can be difficult and costly. In the case of a slug test, the potential exists to push the contaminated groundwater away from the well, possibly contaminating a larger volume of the subsurface. Using In Situ Permeable Flow Sensors requires only very crude estimates of the hydraulic conductivity, alleviating the need for costly pump or slug tests.

Another problem with the standard hydrologic practice is that a relatively large number of boreholes are required to make one velocity measurement. Two holes are required to measure a single component of the flow velocity vector and a minimum of four non-coplanar observation points are required to measure the full three dimensional flow velocity vector. In Situ Permeable Flow Sensors require that only a single hole be drilled to measure the full three dimensional groundwater flow velocity vector.

Yet a third problem with the standard practice is that the velocity determination is an average value characteristic of a broad region whose dimensions are characterized by the separation of the boreholes used for the hydraulic head and hydraulic conductivity measurements. In complex situations, typical of many remediation activities, the groundwater flow velocity may change rapidly over small distances. In Situ Permeable Flow Sensors measure a flow velocity which is an average value within an approximately one cubic meter volume of the subsurface. An additional advantage of the In Situ Permeable Flow Sensors is that it is a simple matter to install a suite of flow sensors and connect their data acquisition systems to a modem so that they can be monitored remotely for extended periods of time without requiring personnel in the field.

There are two other technologies for making direct groundwater flow velocity measurements of which the authors are aware. These are the K-V Geo Flowmeter (Melville et al., 1985; Kerfoot and Massard, 1985; Kerfoot, 1988) and the Colloidal Borescope (Kearl and Case, 1992; 
Kearl, et al., 1993). Both of these technologies rely on measurements made inside a wellbore to measure the horizontal component of the formation flow velocity. The In Situ Permeable Flow Sensor differs from these technologies in that it is deployed directly in the subsurface thereby avoiding borehole effects which can negatively impact the measured velocity magnitude (Melville et al., 1985). In addition, the In Situ Permeable Flow Sensor measures the flow velocity characteristic of a somewhat larger volume $\left(\sim 1 \mathrm{~m}^{3}\right)$ than do the other instruments $\left(\sim 100 \mathrm{~cm}^{3}\right.$ for the Geo Flowmeter and $\sim 1 \mathrm{~mm}^{3}$ for the Colloidal Borescope).

In this report, the theoretical basis for the technology will be outlined and the data processing techniques which have been developed to interpret the data from the flow sensor described. Then the results of a field test at the Savannah River Site in South Carolina will be presented. During this test it was possible to directly compare flow velocity results obtained with the flow sensors to velocities estimated using standard hydrologic techniques.

\section{Principle of Operation}

The basic operating principle of this technology is to bury a thin, cylindrical heater in the ground at the point where the groundwater flow velocity is to be measured. If the heat flux out of the cylinder is uniform over the surface of the cylinder then the temperature distribution on the surface of the cylinder will vary as a function of the direction and magnitude of the groundwater flow velocity past the cylinder. In essence, relatively warm temperatures are observed on the downstream side of the probe and relatively cool temperatures on the upstream side because some of the heat introduced into the formation by the heater is advected around the instrument by fluid flow past the tool.

Romero (in press) derived an expression for the temperature distribution on the surface of a finite length heated cylinder buried in a permeable flow field. The equation is

$$
\begin{aligned}
T(x, z)= & Q R / K[1+R / L P e \cos (\theta-x) \sin \phi] \\
& {\left[\ln \left(2 L / R \sqrt{8^{2}-z^{2}}\right)-R / L P e \cos (\theta-x) \sin \phi\right.} \\
& \left.+\frac{1}{2} \int_{-\delta}^{\delta} \frac{e^{\frac{1}{2} P e[(z-\xi) \cos \phi-|z-\xi|]}-1}{|z-\xi|} d \xi\right]
\end{aligned}
$$

where

$\boldsymbol{T}$ is the temperature at position $\boldsymbol{x}, \boldsymbol{z}$ on the surface of the probe,

$\boldsymbol{x}$ is the angular distance in the horizontal plane from the reference direction to the point on the surface of the cylinder where the temperature is observed,

$z$ is the distance in the vertical direction from the midpoint of the probe to the point on the probe surface where the temperature is observed, made dimensionless by dividing by the half-length of the probe, $\mathrm{L}$,

$\boldsymbol{R}$ is the radius of the probe,

$\boldsymbol{L}$ is the half length of the probe,

$\delta$ is the half length of the heated section of the probe, made dimensionless by dividing by the 
half-length of the probe, $L$

$\boldsymbol{Q}$ is the heat flux per unit area out of the surface of the probe,

$\boldsymbol{K}$ is the thermal conductivity of the fluid-saturated medium,

$P e$ is the Peclet number (see definition below),

$\theta$ is the angular distance in the horizontal plane from the reference direction to the horizontal component of the flow velocity and

$\phi$ is the angle between the vertical direction and the flow velocity vector.

The magnitude of the flow velocity is expressed by the dimensionless Peclet number,

$$
P e=U_{\infty} L \rho c / K
$$

where

$U_{\infty}$ is the magnitude of the Darcy flow velocity far from the probe,

$p$ is the density of the fluid, and

c is the specific heat of the fluid.

The analysis is valid for Peclet numbers up to order 1 . For a typical saturated soil thermal conductivity of $0.01 \mathrm{cal} \mathrm{s}^{-1} \mathrm{~cm}^{-1}{ }^{\circ} \mathrm{C}^{-1}$, this corresponds to a velocity of approximately $0.001 \mathrm{~cm} / \mathrm{s}$ or a few ftday. Extensions of the solution which would make it valid for Peclet numbers up to order 10 are possible (Romero, pers. communication). The analysis further assumes that the cylinder is long and thin $(\boldsymbol{L} / \boldsymbol{R}>\sim 10)$ and that the cylinder is buried in intimate contact with an infinite, saturated, porous medium which is thermally and hydraulically homogeneous and isotropic. Gravitational effects are neglected, meaning that natural convection resulting from heating the water around the probe is assumed to be insignificant. This is valid as long as forced convection is more important than the natural convection induced by warming the water around the probe. The specific criteria to be met is that $P e / R a$ must exceed order 1 where $R \boldsymbol{R}$ is the Rayleigh number given by

$$
R a=\frac{\rho g \beta Q k R L}{K K \mu}
$$

where

$\boldsymbol{g}$ is the gravitational acceleration,

B is the coefficient of thermal expansion of water,

$\boldsymbol{k}$ is the permeability of the medium,

$\boldsymbol{r}$ is the thermal diffusivity of the fluid saturated medium and

$\boldsymbol{\mu}$ is the viscosity of water.

The most important parameters to consider in this case are the magnitude of the flow velocity, $\boldsymbol{U}_{-\infty}$ the heat flux from the probe, $\boldsymbol{Q}$, and the permeability of the medium, $\boldsymbol{k}$. If the flow velocity is low and both the heat flux and the permeability are high, natural convection can be important. For reasonable estimates of all the relevant parameters, natural convection will only be important when the permeability is very high, as might be the case in coarse gravels. In that situation, the importance of natural convection can be evaluated by starting out with a low heat flux from the probe and then increasing the flux and observing any resulting changes in the apparent vertical 
component to the flow velocity.

Figure la illustrates the temperature, calculated according to Equation 1, as a function of azimuth for a probe buried in a horizontal flow field where the groundwater is flowing in a direction toward $90^{\circ}$ from the reference direction on the probe. In the absence of any horizontal flow past the device, the temperature on the surface of the probe is independent of azimuth. If there is a significant horizontal component to the flow velocity, the temperature varies approximately as the cosine of the azimuth with the downstream side of the probe being warmer than the upstream side. Figure $1 \mathrm{~b}$ illustrates the theoretical surface temperature as a function of vertical distance from the vertical midpoint of the probe for a variety of vertically downward flow velocities. If there is no vertical flow past the device, the temperature on the surface of the probe is symmetric about the vertical midpoint of the probe. The vertical midpoint is warmer than the ends of the probe because heat transport away from the ends of a finite length heated cylinder is more efficient than from the mid-section. If the groundwater flow has a significant vertical component, the vertical temperature distribution on the surface of the probe is no longer symmetric about the vertical midpoint of the probe but rather is skewed in the direction of the
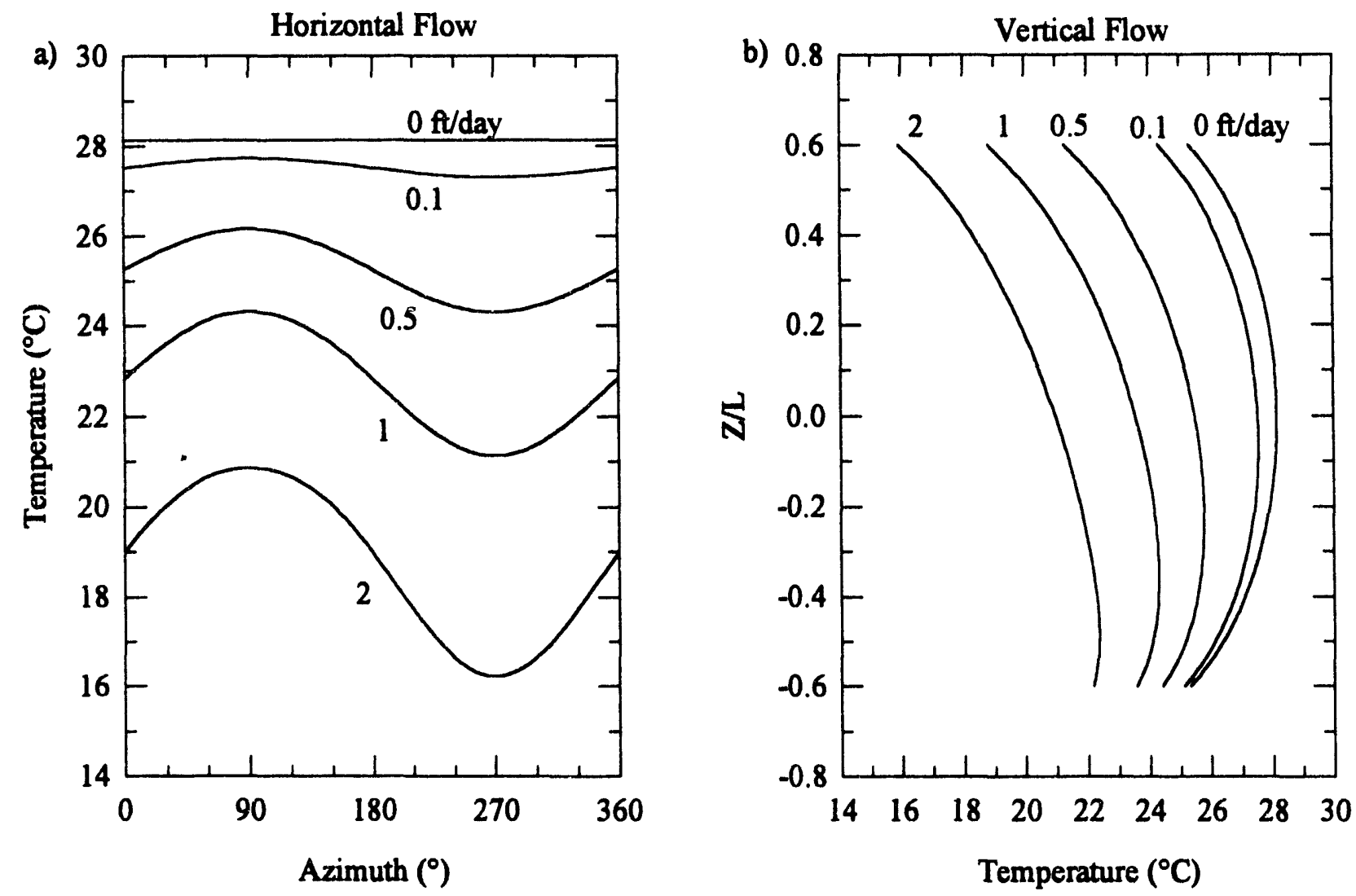

Figure 1 - a) Temperature as a function of azimuth for a probe in a purely horizontal flow field. $270^{\circ}$ and $90^{\circ}$ on the horizontal axis represent the upstream and downstream sides of the probe, respectively. b) Temperature as a function of vertical position for a probe in a downwardly directed flow fieid. 
flow. The downstream end of the probe is warmer than the upstream end. When the flow velocity past the probe has both vertical and horizontal components, the temperature distribution is both asymmetric about the vertical midpoint of the probe and azimuth dependent. Figure 2 shows two contour maps of the theoretical temperature distribution on the surface of a probe, one for a probe buried in a medium with no flow past it and the other for a probe buried in a flow field where there is flow of $1 \mathrm{ft} /$ day oriented in a direction which is toward $90^{\circ}$ from the reference direction and vertically downward $30^{\circ}$ from the horizontal.

In practice, the temperature distribution on the surface of the probe is measured and inverted for the set of flow parameters which results in the best fit, in a least squares sense, between the observed and the theoretical temperature distributions. The inversion scheme used is the SIMPLEX algorithm described by Caceci and Cacheris (1984). A computer code called FLOW has been written to implement the inversion of the data. FLOW, which runs on MS-DOS computers, can also process, graphically display, and otherwise manipulate flow sensor data. The sensors should be sensitive to groundwater flow as low as about $0.01 \mathrm{ft} /$ day, depending on the thermal properties of the medium. They are somewhat more sensitive to the vertical component of the flow velocity than to the horizontal component because of their long aspect ratio.

Confidence limits on the flow parameters which best fit the available data are determined using a Monte Carlo technique outlined in Press et al. (1986). They are calculated as follows: first a "best fit" set of flow parameters is calculated. The root mean square error between the data and the model is calculated according to the formula

$$
E_{r m s}=\sqrt{\frac{\sum_{i=1}^{N}\left(T_{i, \text { model }}-T_{i \text { data }}\right)^{2}}{N-1}}
$$

where $\boldsymbol{E}_{\mathrm{m} \text { s }}$ is the root mean square error, $\boldsymbol{N}$ is the number of temperature observations used in the analysis and $T_{i \text { mode }}$ and $T_{i, m}$ are the model and data temperature values at the $t^{\text {th }}$ observation location, respectively. Then $N$ random temperature values are drawn from a normally distributed probability distribution which hrs a mean of zero and a standard deviation of $\boldsymbol{E}_{\boldsymbol{r m s}}$. These $\boldsymbol{N}$ temperature values are added to the original $N$ temperature values used in the analysis and another set of flow parameters is calculated based on this new, "noisy" temperature distribution. This procedure is repeated $m$ times, each time adding a different set of $N$ random temperature deviations to the original temperature distribution. In this way $m$ different sets of flow parameters are generated. The $95 \%$ confidence limits on the original set of flow parameters, calculated using the undisturbed temperature distribution, is equal to twice the standard deviation of the $m$ sets of flow parameters calculated using the "noisy" temperature distributions. Note that $m$ needs to be a fairly large number, like 100 or more, in order to predict the $95 \%$ confidence limits with a reasonable degree of accuracy.

The way in which the probes are fabricated and deployed in the ground is critical to obtaining a valid measurement (Figure 3). The current strategy involves constructing very simple, inexpensive probes that can be permanently buried in saturated, unconsolidated sediments. Each probe consists of a rod of low thermal conductivity, closed cell, polyurethane foam, 30 inches long by 2 inches in diameter, surrounded by a thin film, flex circuit heater, an array of 30 carefully calibrated thermistors and a waterproof jacket. The design seeks to achieve a high degree of uniformity of the heat flux from the surface of the probe and sufficient temperature 

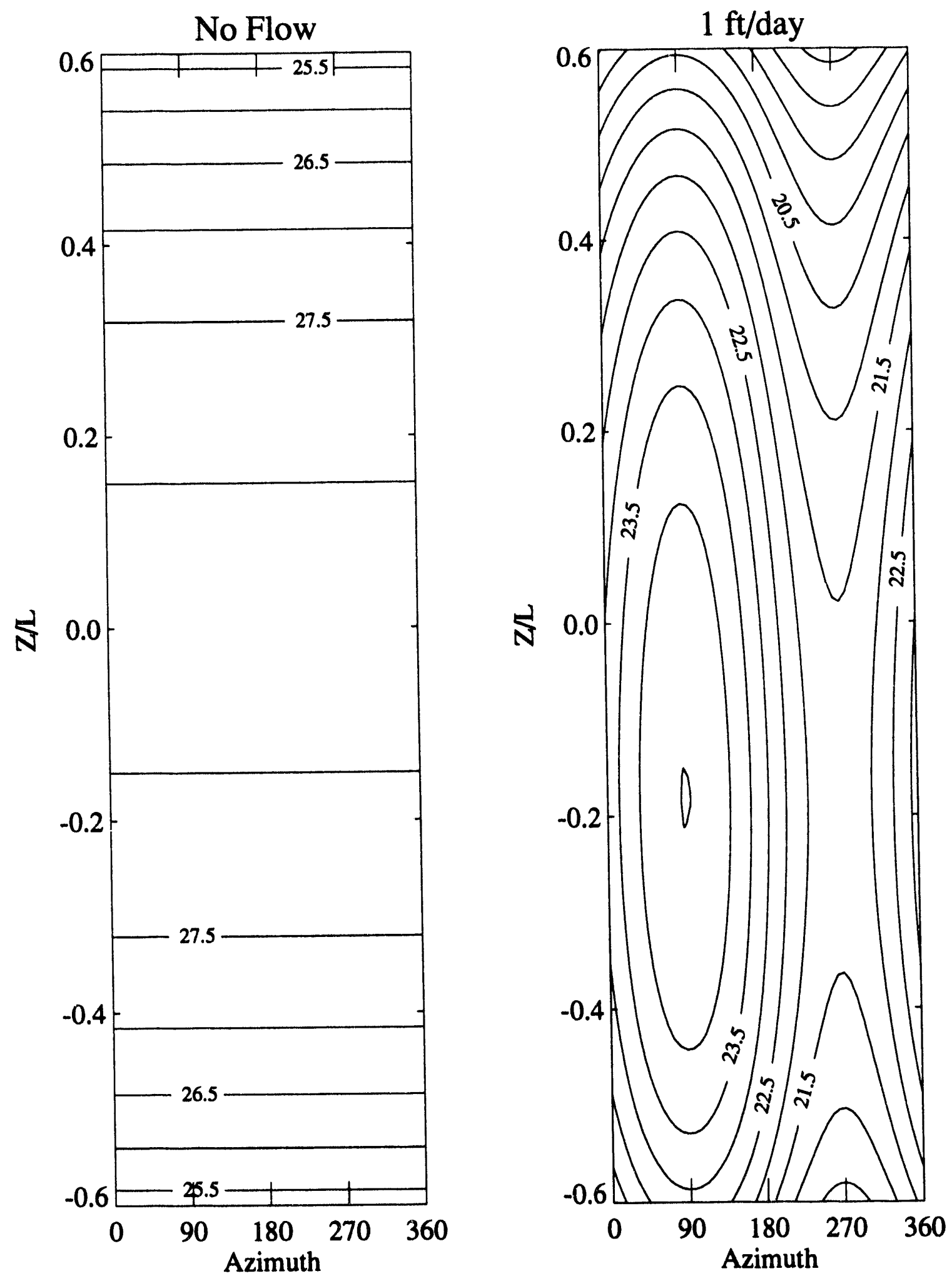

Figure 2 - Contour maps of the theoretical probe surface temperature for a) a flow sensor buried in a medium where there is no flow and b) where there is flow of $1 \mathrm{ft} /$ day oriented in a direction which is toward $90^{\circ}$ from the reference direction and $30^{\circ}$ down from the horizontal. 


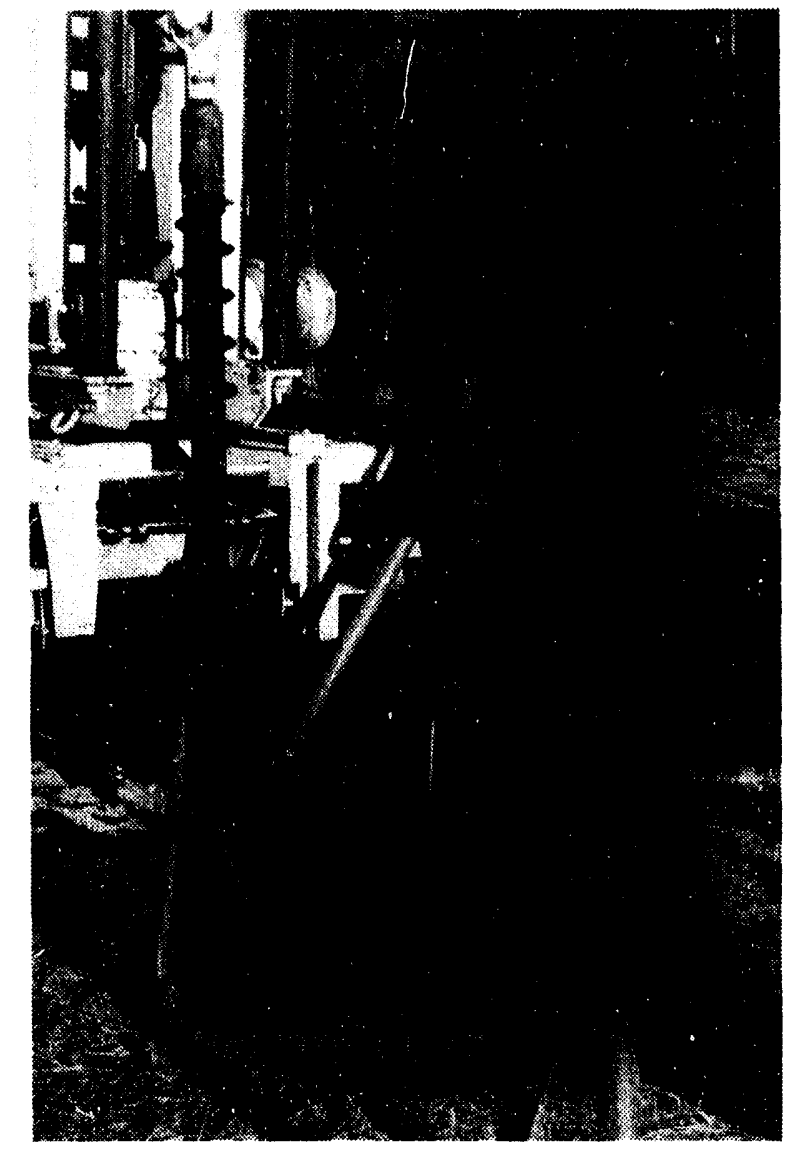

Figure 3 - An In Situ Permeable Flow Sensor being emplaced into the subsurface through a hollow stem auger.

sensors near the surface of the probe to adequately characterize the temperature distribution on its surface. The pattern of the thermistor distribution near the surface of the probe is illustrated in Figure 4.

At the deployment site, sufficient 1 inch diameter, schedule 80 PVC pipe is attached to the top of the probe to reach to the ground surface after the probe is deployed in the ground. Marks are made along the PVC pipe so that the horizontal orientation of the probe will be known after the probe is emplaced. While more sophisticated techniques for determining the horizontal orientation of the probes exist, this simple, inexpensive process has worked well in experiments to date.

To emplace the probes, a hollow stem auger is used to drill to the depth where the measurement is to be made, the probe is lowered down the center of the auger and the auger is retracted from the hole, leaving the probe in the ground. In saturated, unconsolidated formations, the hole collapses around the probe, leaving it in a relatively undisturbed setting. Although the probes cannot be recovered after being deployed in this way, they can continue to provide flow measurements for extended periods of time. They should last 1 to 2 years; until the waterproof 
coatings ultimately leak. The principal advantage of this strategy is that the probe is not deployed in a hole where the flow can be significantly different from the flow in the surrounding formation (Melville et al., 1985; Kerfoot, 1988; Wheatcraft and Winterberg, 1985).

\section{A Typical Flow Sensor Data Set}

When a velocity measurement is to be obtained, the heater on the flow sensor is activated. Typically, 120 watts of DC power is continuously supplied to the probe. Figure 5a illustrates the temperature of the 30 thermistors on the probe surface as a function of time. Before activation of the heater, the average temperature of all the thermistors shown in Figure 5a was $19.48 \pm .03^{\circ} \mathrm{C}$, the equilibrium temperature of the ground at this location. After activation of the heater, the temperature of the probe surface rose abruptly and reached a quasi steady state after about 24 hours. Note that the temperature of some thermistors rose more quickly than others, indicating a change in the relative temperature distribution on the surface of the probe. Figure $5 \mathrm{~b}$ illustrates the deviation from the average surface temperature of the probe, as a function of time. The data plotted in Figure 5b were calculated by first determining the average temperature of the 30 thermistors at each time step, and then subtracting that value from all of the individual temperature readings, at that same time step. Before the heater was activated, the deviations from the average temperature are all very small, less than a few hundredths of a degree. Within the first half hour after the heater was activated the magnitudes of the deviations from the average temperature increase abruptly, to about $\pm 0.6^{\circ} \mathrm{C}$. After that the relative temperatures continue to change, albeit more slowly, until the relative temperatures ultimately reach equilibrium after about 15 or 20 hours.

Two thermal behaviors are evident in the time history of the relative temperature distribution on the surface of the probe illustrated in Figure 5b. A short time behavior is observed for the first half hour or so after the activation of the heater and a long time behavior after that. The short time behavior is related to the establishment of the thermal gradients within the probe itself while the long term behavior reflects the overall geometry of the probe, the thermal properties of the material surrounding the probe and the direction and magnitude of the fluid flow past the probe. The long term behavior is the signal to be analyzed while the short term behavior acts to corrupt the signal of interest and must be removed from the data.

Several factors contribute to the short term signal, the most significant of which is that the thermistors are not actually measuring the temperature at the surface of the probe. In order to isolate the thermistors from the water surrounding the probe and protect them from the rigors of emplacement into the subsurface, the thermistors are located about $1 \mathrm{~mm}$ below the surface of the probes. Given the heat flux out of the probe of about $0.0272 \mathrm{cal} \mathrm{s}^{-1} \mathrm{~cm}^{-2}$, the temperatures measured by the thermistors can be expected to be on the order of $1^{\circ} \mathrm{C}$ higher than the temperature at the surface of the probe adjacent to the thermistors. In addition, the temperature difference between the temperature at the thermistor locations and the surface of the probes can be expected to vary from place to place on the surface of the probe by as much as a few tenths of a ${ }^{\circ} \mathrm{C}$, due primarily to differences in thickness of the material separating the thermistors from the surface of the probe. Other factors which would influence the magnitude of these short term variations would be small spatial variations in the heat flux from the probe, uneven heating of

the foam from which the probe is fabricated and variations in thermal properties of all of the materials in the vicinity of the thermistors, including the foam, the heater and thermistor circuitry, the waterproof sealant and the geologic material in which the probe is buried. 

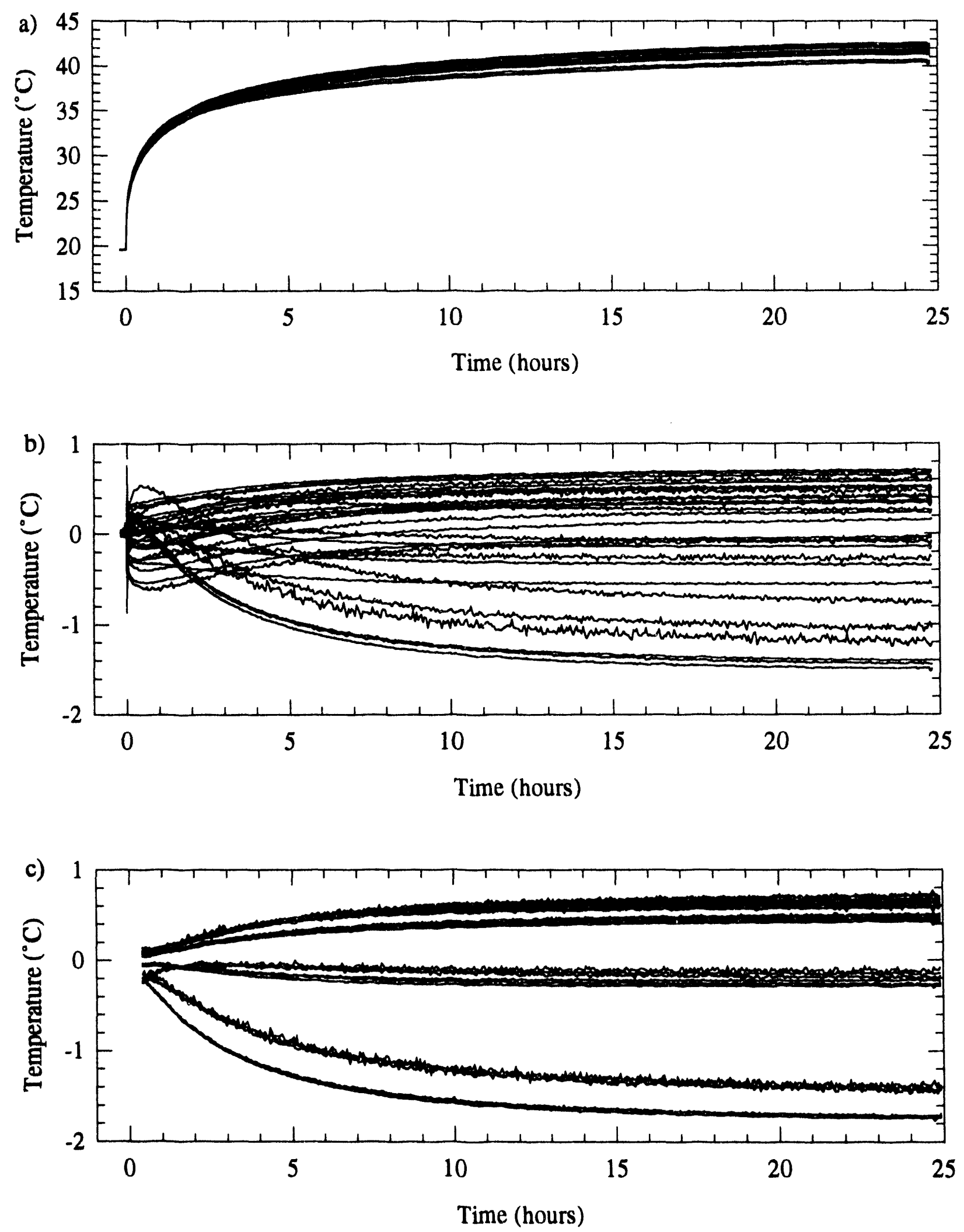

Figure 5 - a) Temperature on the surface of the probe, b) deviation from the average temperature and c) corrected deviation from the average temperature as a function of time. 
Fortunately, these short term effects can be readily corrected for by using the long term temperature trends. The procedure is illustrated in Figure 6, which portrays the deviation from the average probe surface temperature vs time for one thermistor on the probe. To remove the effect of the short term temperature variations, the long term temperature behavior is extrapolated backward to the time of activation of the heater. With the data in Figure 6, this is accomplished by fitting a $2^{\text {nd }}$ order polynomial to the temperature data in the time range from 0.5 to 3.5 hours after the time of heater activation and extrapolating that trend back to time 0 . The extrapolated temperature at time 0 is the correction factor which is subtracted from all the data obtained from that thermistor. The set of 30 correction factors for a particular probe must be determined after the probe is emplaced in the ground since the thermal properties of the material surrounding the probes influence the magnitude of the correction factors. Once a set of correction factors has been determined for a particular probe, they can be applied to that probe for all subsequent data sets collected with that probe as long as the heater power output remains the same. The FLOW computer program provides capabilities to facilitate the determination, storage, retrieval and application of these correction factors.

The result of applying these correction factors to the data in Figure 5b is shown in Figure 5c. These data indicate that after activation of the heater, the relative temperature distribution on the surface of the probe changes, with temperatures at some thermistor locations increasing while those at other locations are decreasing, relative to the average temperature of the surface of the probe. The temperature distribution reaches a steady state condition after about 24 hours.

Figure 7 illustrates the temperature distribution on the surface of the probe at the end of the data set illustrated in Figure 5c. The symbols in Figures $7 \mathrm{a}$ and $7 \mathrm{~b}$ represent the measured temperature on the surface of the probe as a function of azimuth and vertical position along the probe, respectively. The temperature as a function of azimuth has a peak to peak amplitude of only about $.06^{\circ} \mathrm{C}$, indicating a small horizontal component to the flow velocity. The temperature as a function of vertical position on the probe is relatively symmetric about the vertical midpoint of the probe, suggesting that the vertical component of the flow is relatively small as well. Inversion of the temperature data yields a best fit set of flow parameters with a horizontal component of flow of $0.02 \pm 0.01 \mathrm{ft} /$ day oriented in a direction which is $250^{\circ} \pm 55^{\circ}$ from the reference direction of the probe. The vertical component of the flow velocity vector determined from the data in Figure $5 \mathrm{c}$ is $0.05 \pm 0.01 \mathrm{ft} /$ day. The theoretical temperature distribution on the surface of the probe calculated using these flow velocity parameters is illustrated in Figure 7 with the smooth curves.

These flow velocity levels are representative of the lowest flow velocities resolvable by the flow sensors. In this context, the lowest velocities that can be measured are defined as velocities 

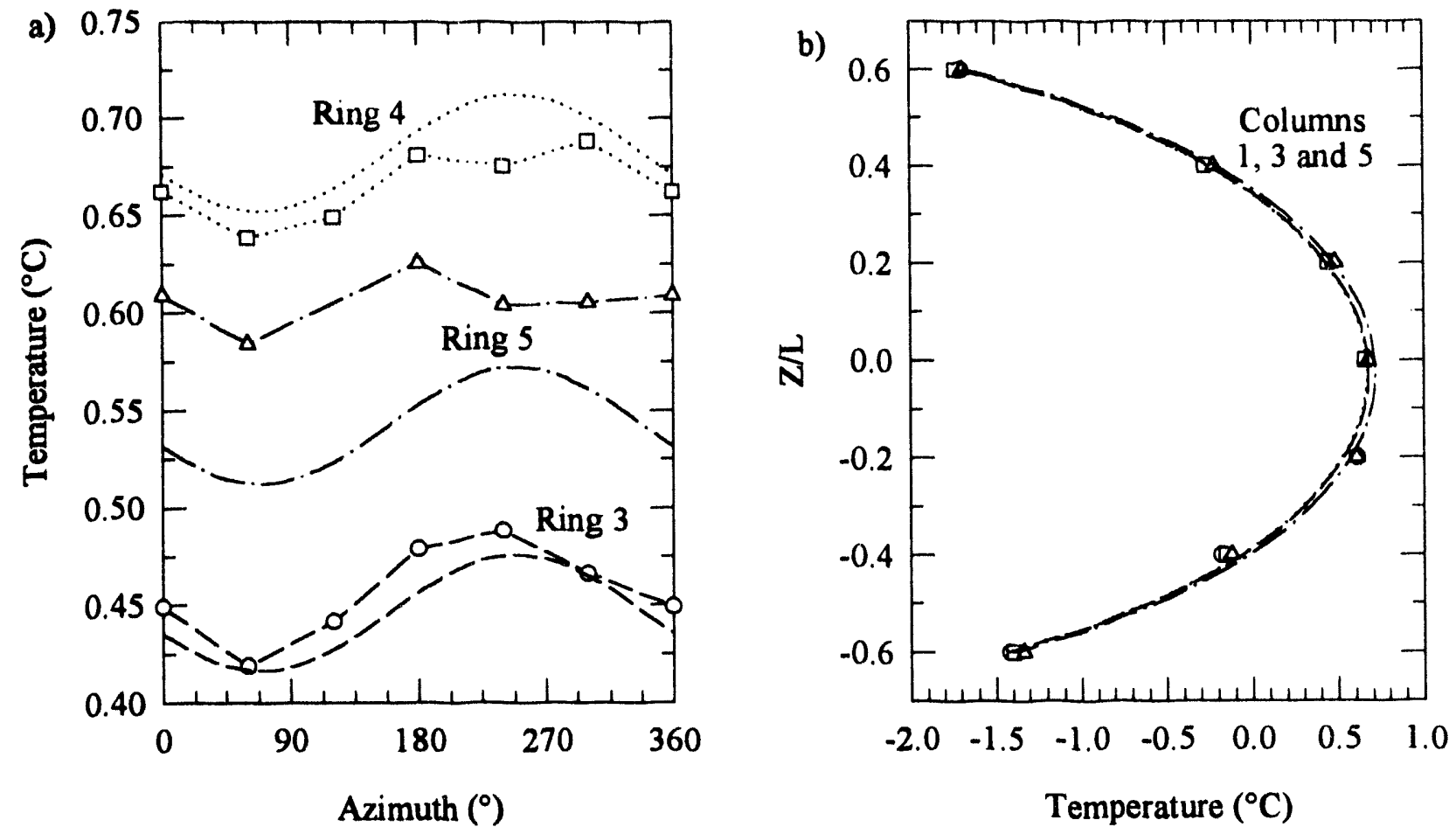

Figure 7 - Temperature as a function of a) azimuth and b) vertical position on the probe surface. The points are data and the smooth curves are theoretical fits to the data.

that are so low that the uncertainty in the magnitude of the flow velocity is as large as the velocity itself. Recall that the uncertainty in the velocity is dependent on the difference between the measured and best-fit theoretical temperature distributions as described by Equation 4 . Factors that contribute to the discrepancy between the theoretical and observed temperatures are the accuracy of the thermistor calibrations and errors in the determination of the correction factors which are applied to the observed temperatures as described above. The thermistors are calibrated such that temperature differences between thermistors are probably accurate to within $\pm 0.01^{\circ} \mathrm{C}$. The errors introduced during the calculation of the correction factors are probably larger than that, on the order of 0.05 to $0.1^{\circ} \mathrm{C}$, so the accuracy of the correction factors is probably the main limitation in terms of the lowest flow velocities that can be resolved.

\section{Field Calibration Experiment}

To ascertain the validity of the flow sensor measurements, a field test was conducted in which it was possible to compare flow velocities observed with the flow sensors directly with measurements obtained simultaneously with standard hydrologic techniques. The test was conducted at the Savannah River Site in South Carolina. The location of the test is underlain by a 46 foot thick leaky aquifer consisting of unconsolidated sandy sediments (Figure 8). The aquifer is bounded above and below by relatively impermeable clay layers. Four wells penetrate the aquifer at the site. Each well is screened throughout the entire vertical extent of the aquifer. For this experiment, a pump was placed in P26AP and pressure transducers were deployed in P26M1, P26M2 and P26AI. The latter three wells are at distances of 15.5, 40 and 50 feet from 

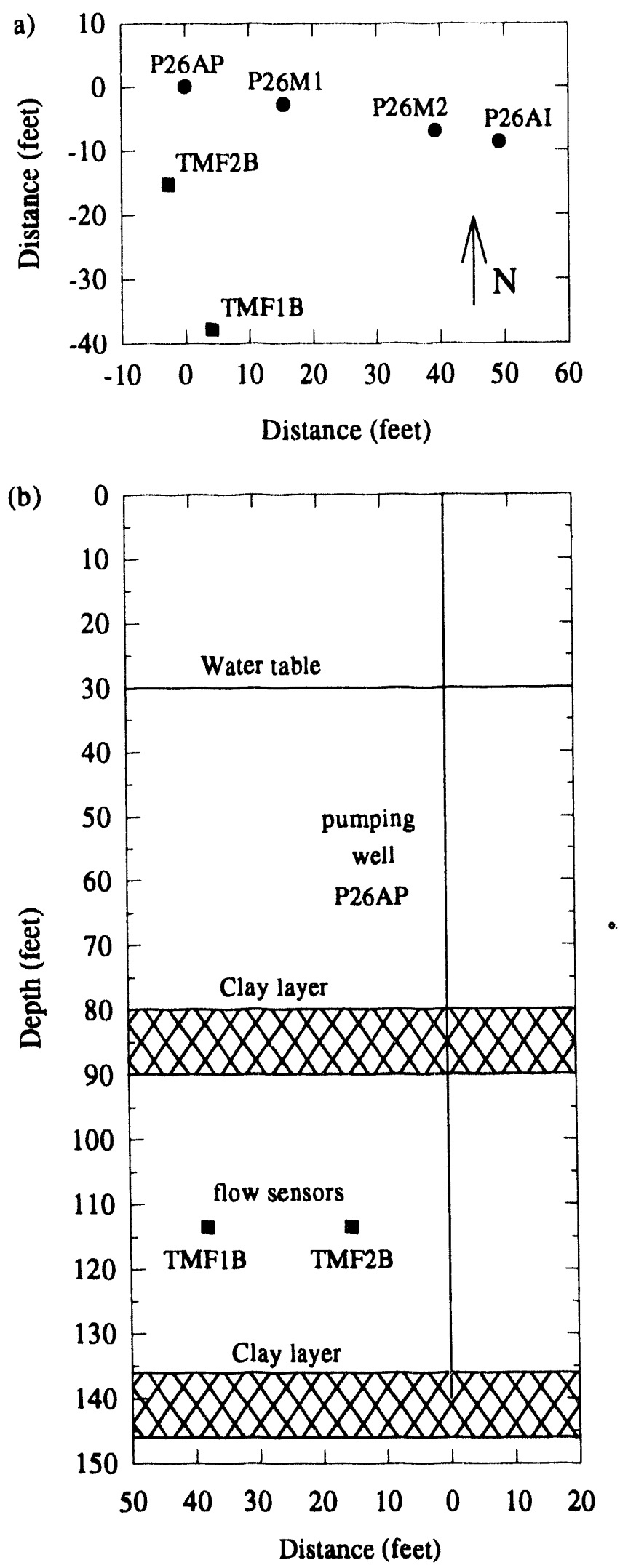

Figure 8 - a) Map and b) cross section of the field test site at Savannah River. 
P26AP. The experiment was conducted in two stages. During the first stage, P26AP was pumped at a rate of 15.5 gallons per minute (gpm) for about 24 hours and then at $9 \mathrm{gpm}$ for about 60 additional hours. During the second stage, which took place about 3 weeks after the first stage, P26AP was pumped at $6 \mathrm{gpm}$ for 60 hours and then at $3 \mathrm{gpm}$ for 100 hours. The drawdown in the three piezometer wells was observed throughout the period of time that P26AP was being pumped and for several days after pumping ceased. Two In Situ Permeable Flow Sensors, TMF1B and TMF2B, were placed at a depth of 113.5 feet and distances of 38 and 15.5 feet from P26AP. These flow sensors were activated approximately 24 hours before each pumping test began, were monitored throughout each test and for 1 to 2 days after the pump had been turned off.

Figures $9 \mathrm{a}$ and $9 \mathrm{~b}$ illustrate the temperature and the corrected deviation from the average temperature, as a function of time, measured by the thermistors on the flow sensor which was closest to the pumping well during the time that P26AP was being pumped at 6 and then $3 \mathrm{gpm}$. The data indicate that significant changes in the temperature distribution on the surface of the probe occurred in response to changes in the pumping rate. The nature of these changes in the surface temperature distribution are illustrated in Figure 9c which shows the temperature as a function of azimuth around the probe for the data from thermistor ring \#3 at 0,3,6,9 and 15 $\mathrm{gpm}$. The data indicate that when the pump was active, the side of the probe closest to the pumping well (zero azimuth in Figure 9c) was warmer than the side furthest from the pumping well ( $180^{\circ}$ in Figure $\left.9 \mathrm{c}\right)$ and that the amplitude of the temperature variations around the probe increased with the pumping rate.

Figure 10 illustrates the full three dimensional flow velocity, as a function of time, that results in the best fit between the measured temperatures illustrated in Figure $9 \mathrm{~b}$ and theoretical temperatures calculated according the Equation 1. In Figure 10b, zero azimuth refers to the direction from the flow sensor to the pumping well. This corresponds to the expected flow direction when the pump was active. Prior to time 0 , the time when the pump in P26AP was activated, the magnitude of the horizontal component of the flow velocity was approximately $0.04 \pm 0.03 \mathrm{ft} /$ day, and was oriented in a direction of about $-135^{\circ} \pm 55^{\circ}$ relative to the direction to the pumping well. Figure 10c illustrates the magnitude of the vertical component of the flow velocity, which prior to activation of the pump was approximately $0.11 \pm 0.02 \mathrm{ft} /$ day. The relative magnitudes of the vertical and horizontal components of the flow velocity indicate a flow velocity vector orientation of $70^{\circ} \pm 15^{\circ}$ with respect to the horizontal plane. In other words, the pre-test flow was directed in a predominantly vertically upward direction.

When the pump in P26AP was activated at time zero, the horizontal component of the flow velocity increased immediately and reached a quasi-equilibrium value of about $0.50 \pm 0.075 \mathrm{ft} /$ day after about 30 hours. The orientation of the horizontal component of the flow velocity changed to about $2^{\circ} \pm 7^{\circ}$ relative to the direction to the pumping well. The vertical component of the flow velocity went from positive (vertically upward) to negative (downward). The flow velocity vector was oriented approximately $-22^{\circ} \pm 5^{\circ}$ from the horizontal. Approximately 3 days after pumping began, the pumping rate was reduced from $6 \mathrm{gpm}$ to $3 \mathrm{gpm}$. The horizontal component of the flow velocity dropped to about $0.25 \pm 0.04 \mathrm{ft} /$ day and the orientation changed to about $8^{\circ} \pm 8^{\circ}$ relative to the direction to the pumping well. The vertical component changed to $0.01 \pm 0.02 \mathrm{f} /$ day, indicating that the flow velocity was essentially horizontal.

Approximately one week after pumping began, the pump in P26AP was turned off and the horizontal component of the flow velocity declined to approximately zero. The uncertainty in 

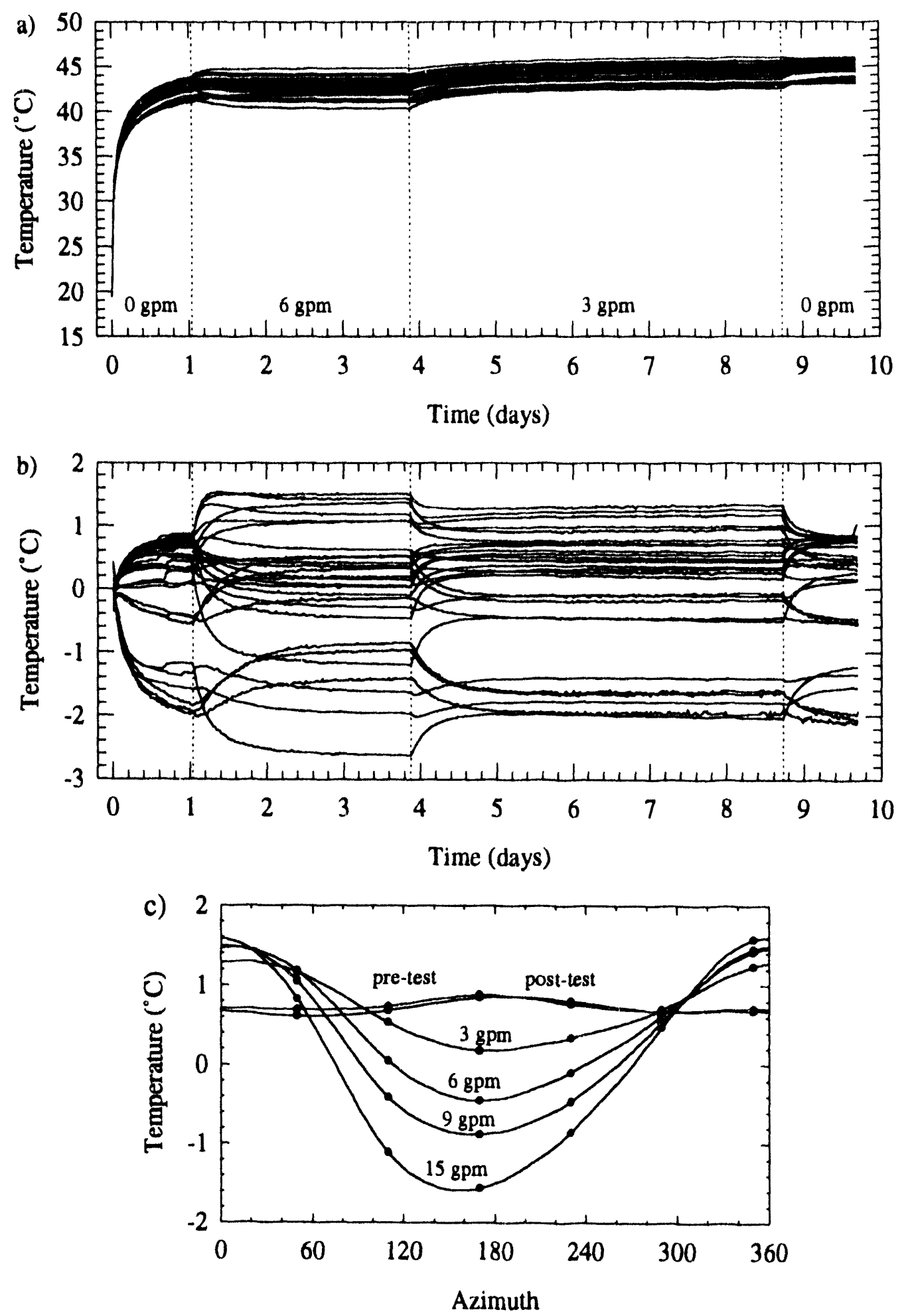

Figure 9 - a) Temperature and b) corrected deviation from the average temperature on the surface of the probe in TMF2B $(\mathrm{R}=15.5 \mathrm{ft})$ at 6 and $3 \mathrm{gpm}$. Time 0 is the time of activation of the heater on the probe. The vertical dotted lines indicate the times when changes in the pumping rate were instituted. c) Temperature as a function of azimuth for the 6 thermistors on ring 3 . The symbols represent the data and the curves are cubic spline fits to the data. 
a)

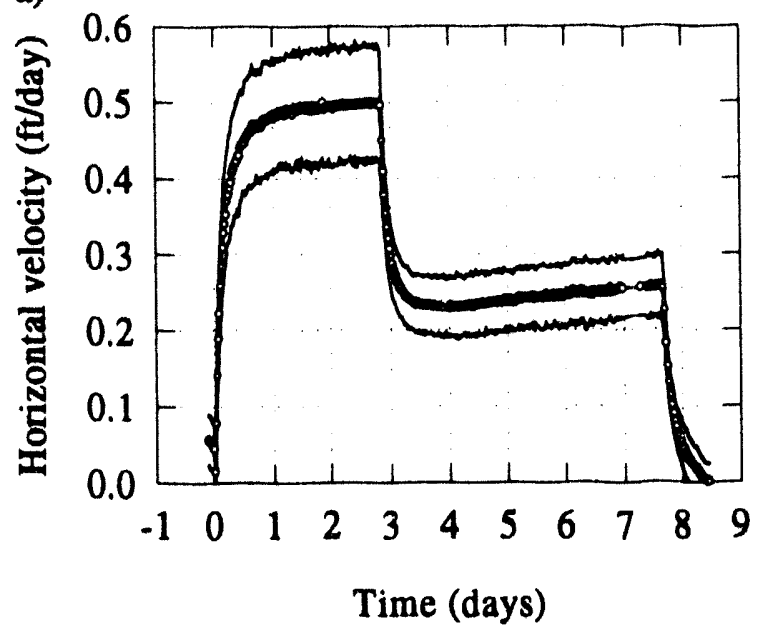

c)

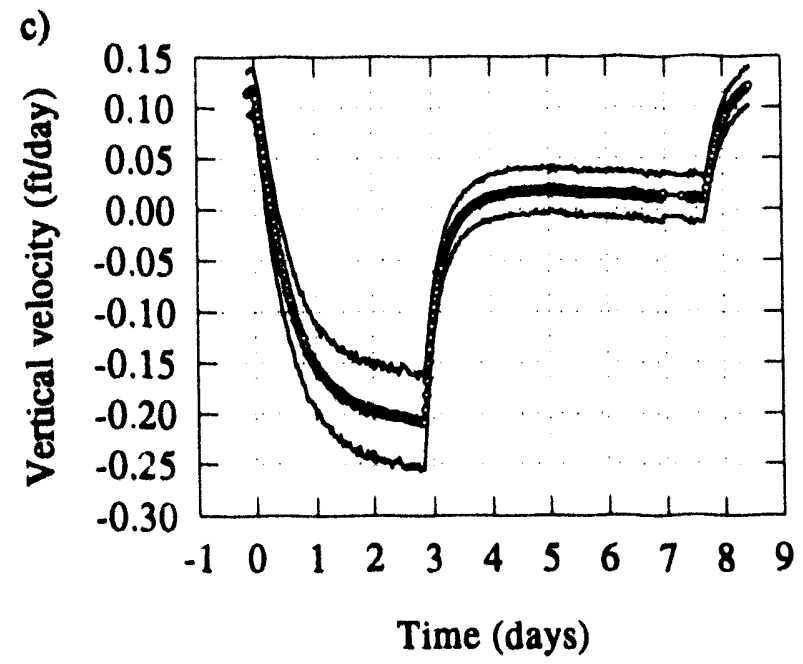

b)

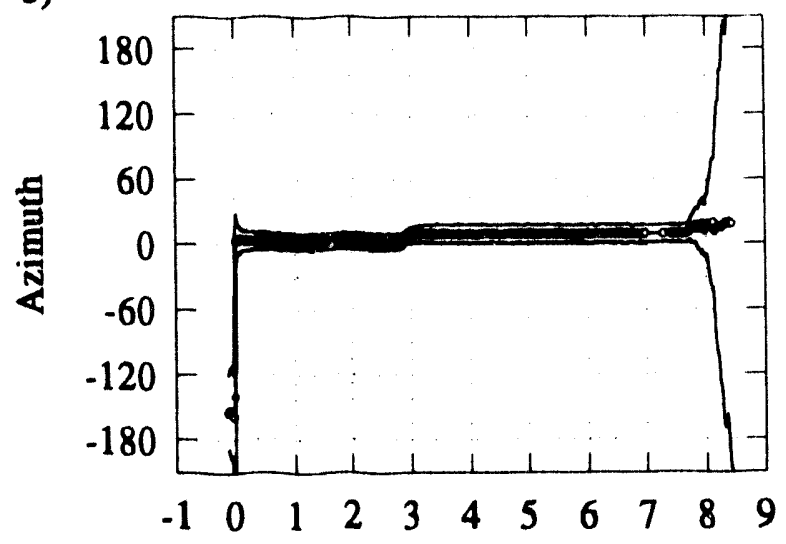

Time (days)

d)

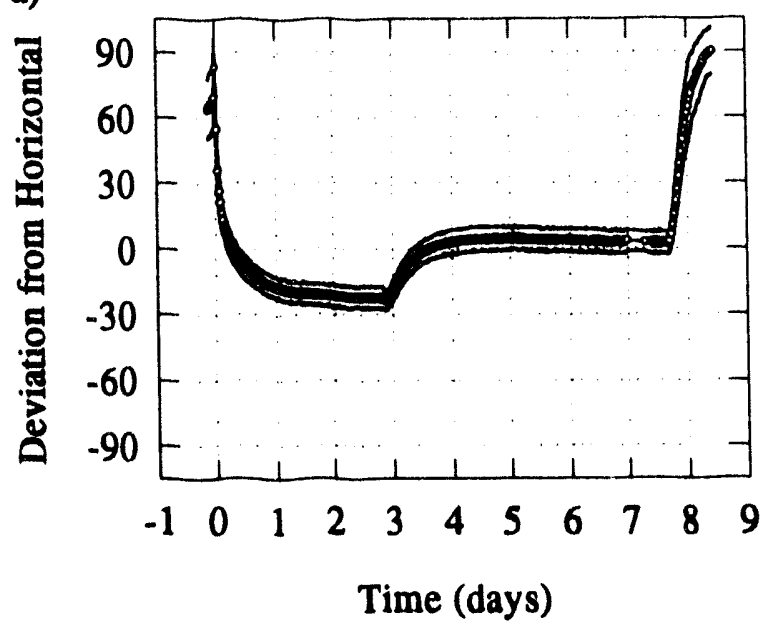

Figure 10 - Flow velocity measured by the probe in TMF2B $(R=15.5 \mathrm{ft}$ ) when the pumping rate was 6 and then $3 \mathrm{gpm}$. Time 0 is the time of activation of the pump which was approximately one day after activation of the heater on the probe. 
the orientation of the horizontal component of the flow velocity increased to $\pm 180^{\circ}$, implying that the direction of the horizontal component of the flow velocity is undetermined. This increase reflects the fact that the orientation of the horizontal component of the flow velocity can only be estimated with very large uncertainty when the magnitude of the horizontal component of the flow velocity is very small. The vertical component of the flow velocity returned to being vertically upward, as it was before pumping began.

Figures 11,12 and 13 illustrate the velocities observed at TMF2B $(R=15.5 \mathrm{ft})$ at 15 and 9 gpm, at TMF1B ( $R=38 \mathrm{ft})$ at 6 and $3 \mathrm{gpm}$ and at TMF1B $(\mathrm{R}=38 \mathrm{ft})$ at 15 and $9 \mathrm{gpm}$, respectively. The general pattern of the flow velocities illustrated in these figures is basically the same as the flow velocity observed in TMF2B at 6 and $3 \mathrm{gpm}$ except that the magnitudes of the vertical and horizontal components of the flow velocities increase with both pumping rate and proximity to the pumping well.

The flow velocities measured by the two probes as a function of pumping rate are plotted in Figure 14. Figures $14 \mathrm{a}, \mathrm{b}$ and $\mathrm{c}$ illustrate the flow velocity in cylindrical coordinates and Figure $14 \mathrm{~d}$ is a plot of the vertical angle between the three dimensional flow velocity vector and the horizontal plane. The data plotted in Figure $14 \mathrm{a}$ and $14 \mathrm{~b}$ at a pumping rate of zero reflect the measured background flow velocity while the data shown in those plots at non-zero pumping rates have had the background flow subtracted from them in order to isolate the effect of pumping on the flow velocity. The lines in Figure 14a are linear regression fits to the data collected when the pump was active, extrapolated back to a pumping rate of zero. Both measured and corrected flow velocities are tabulated in Tables 1 and 2. The magnitudes of the horizontal and vertical components of the flow velocity from the far probe (TMF1B, $R=38 \mathrm{ft}$ ) at 6 and $15.5 \mathrm{gpm}$ are not included in Figure 14 because the probe had not entirely reached equilibrium by the time the pumping rate was changed during the two phases of the experiment. If the trends of the data in Figures $12 \mathrm{a}$ and $13 \mathrm{a}$ are extrapolated to equilibrium values, the extrapolated points would fall very close to the line labeled $38 \mathrm{ft}$ in Figure 14a.

Before pumping began, the near and far probes indicated horizontal flow velocities of $0.04 \pm 0.03 \mathrm{ft} /$ day and $0.02 \pm 0.01 \mathrm{ft} /$ day, respectively. In both cases, these velocities are near the lower limit of the velocity that the probes can detect, as is evident from the fact that the uncertainties are nearly as large as the velocity values themselves. The azimuths of the near and far probes are $-135^{\circ} \pm 55^{\circ}$ and $-59^{\circ} \pm 44^{\circ}$, respectively. These azimuths are measured relative to the direction toward the pumping well. In geographic coordinates, these directions are $-125^{\circ} \pm 55^{\circ}$ and $-65^{\circ} \pm 44^{\circ}$, indicating that the background flow at the site is in a basically westerly direction, albeit with large uncertainty. This direction corresponds reasonably well with the expected direction, toward the Savannah River, which is about $1 / 2$ mile away in a westsouthwesterly direction.

Prior to the initiation of pumping, both flow sensors observed significant vertical components of flow, dominating the horizontal components in both cases, but of opposite signs (Figures 14c and $\mathrm{d}$ ). The near probe measured a vertically upwardly directed flow while the far probe measured a downwardly directed flow. There are no convincing hydrologic explanations for the measured background vertical flow velocities. The measured signals may be influenced by vertical variations in thermal properties of the material surrounding the probes which were not completely accounted for in the determination of the probe correction factors described earlier. It is possible, but cannot be confirmed, that these vertical variations in thermal properties were introduced during emplacement of the probes into the subsurface. Since these measurements of the background vertical flow velocities are not well understood, they have not been subtracted 

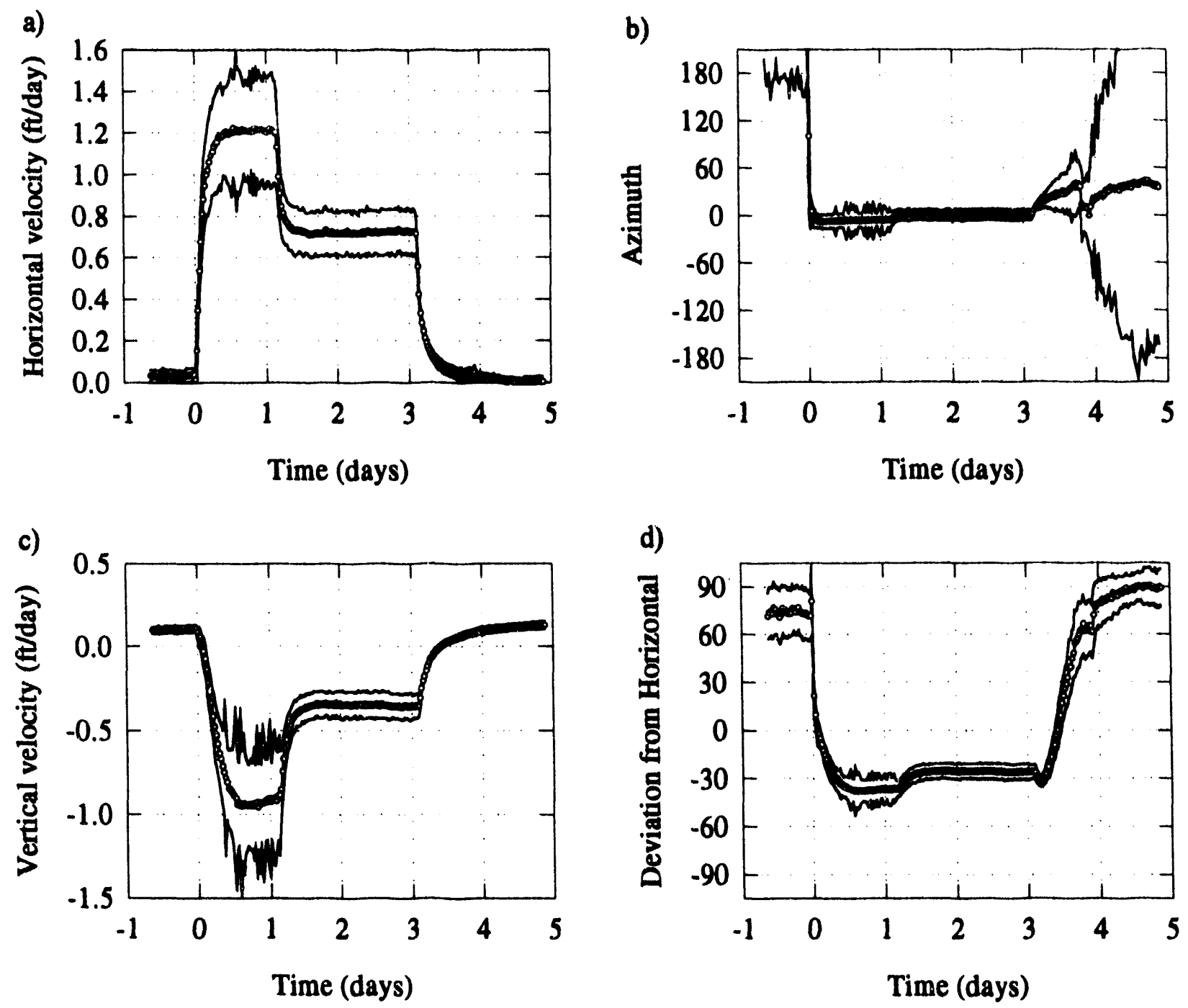

Figure 11 - Flow velocity measured by the probe in TMF2B $(R=15.5 \mathrm{ft})$ when the pumping rate was 15.5 and then $9 \mathrm{gpm}$. Time 0 is the time of activation of the pump which was approximately one day after activation of the heater on the probe. 
a)
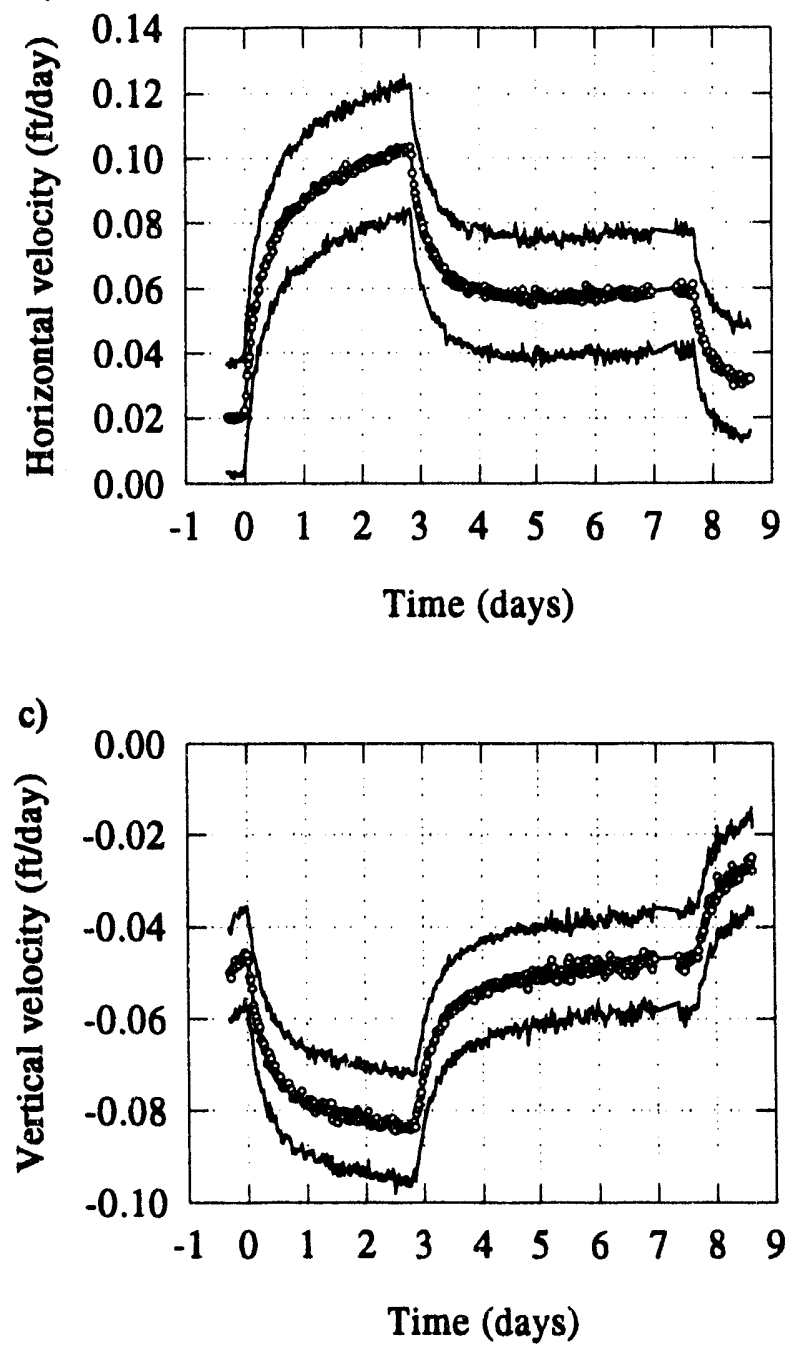

b)

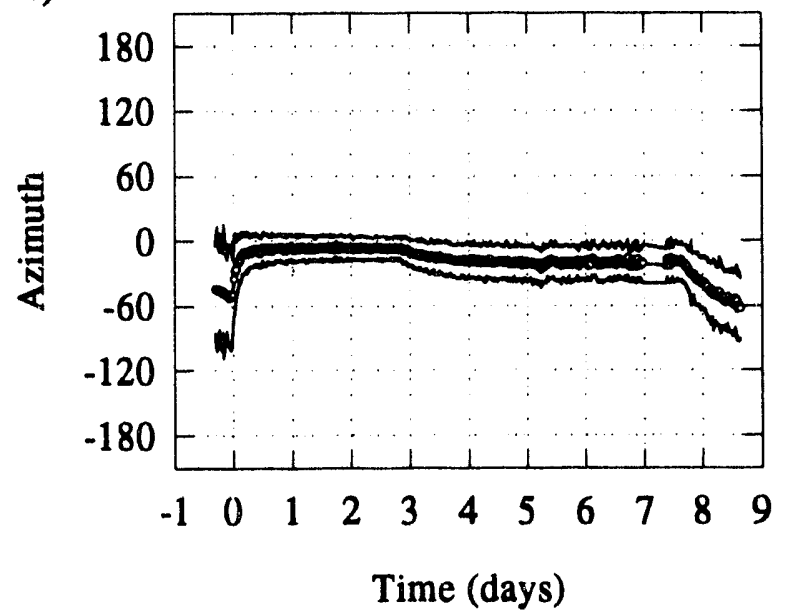

d)

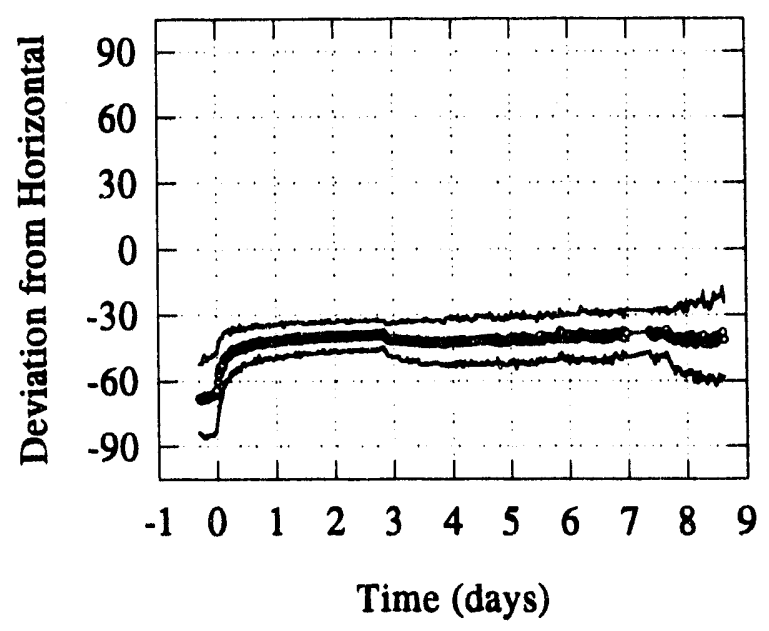

Figure 12 - Flow velocity measured by the probe in TMF1B $(R=38 \mathrm{ft})$ when the pumping rate was 6 and then $3 \mathrm{gpm}$. Time 0 is the time of activation of the pump which was approximately one day after activation of the heater on the probe. 

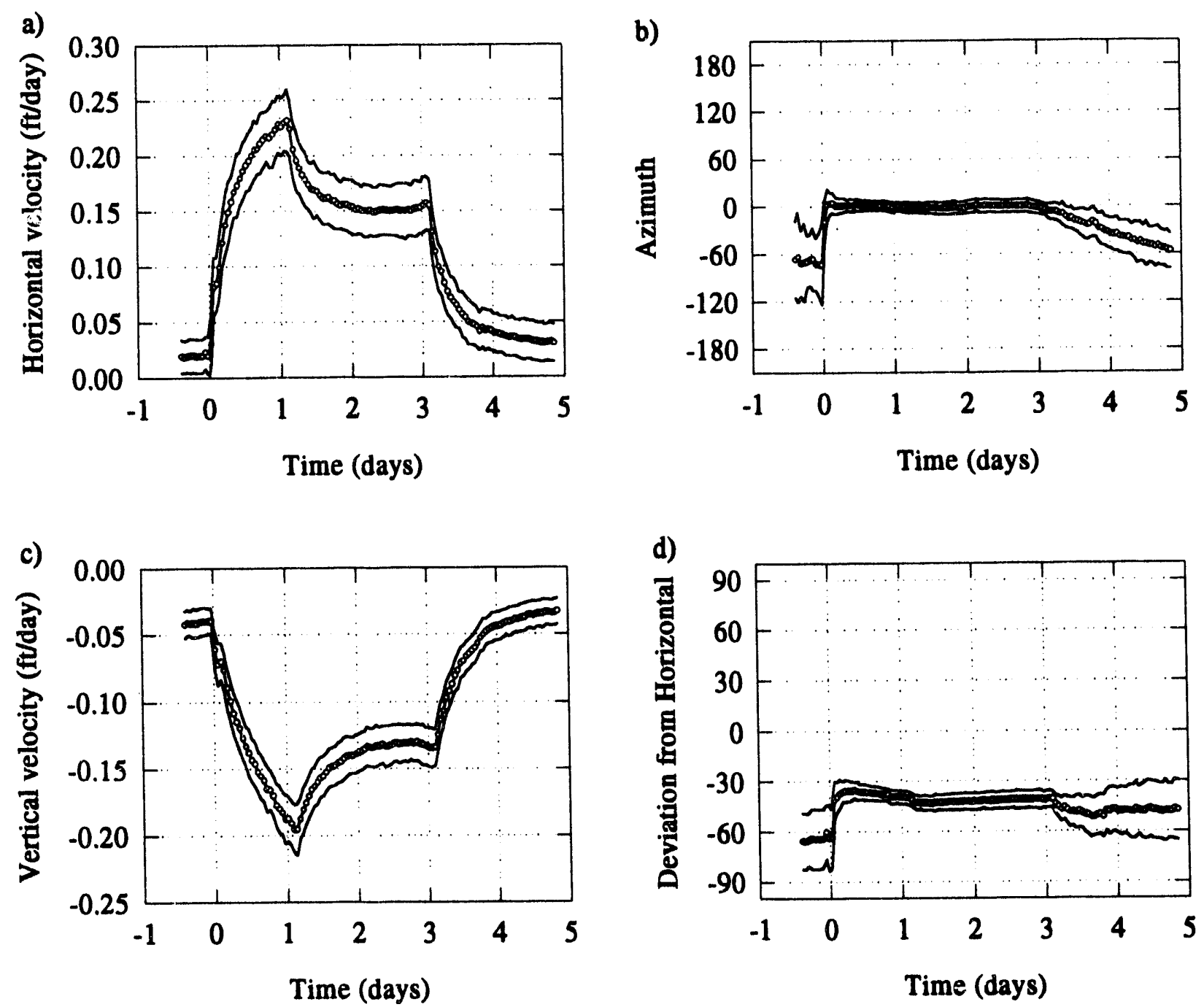

Figure 13 - Flow velocity measured by the probe in TMF1B $(R=38 \mathrm{ft}$ ) when the pumping rate was 15.5 and then $9 \mathrm{gpm}$. Time 0 is the time of activation of the pump which was approximately one day after activation of the heater on the probe. 
a)

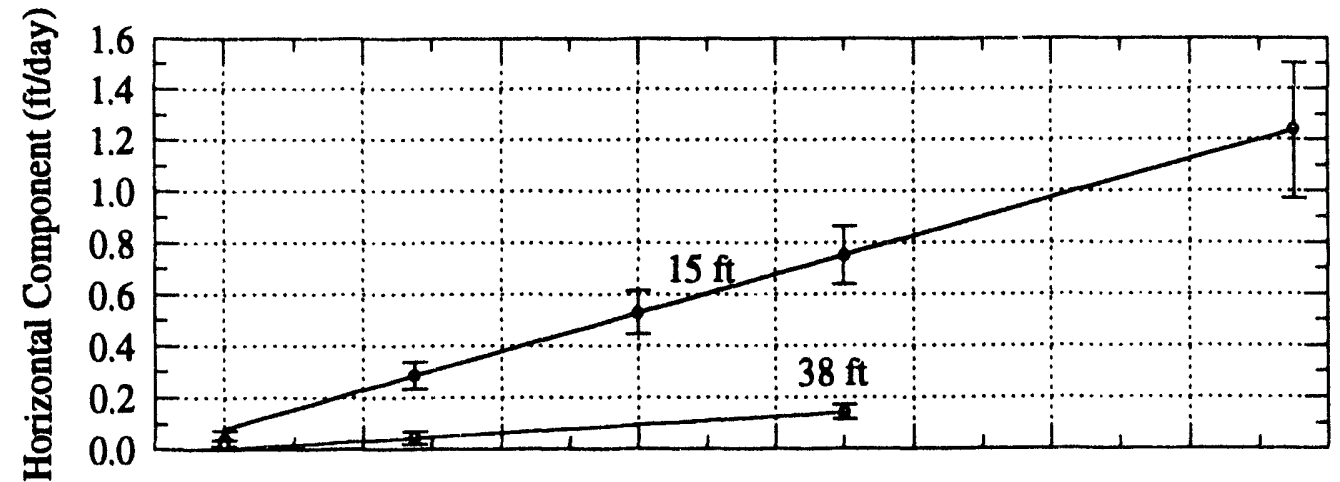

b)

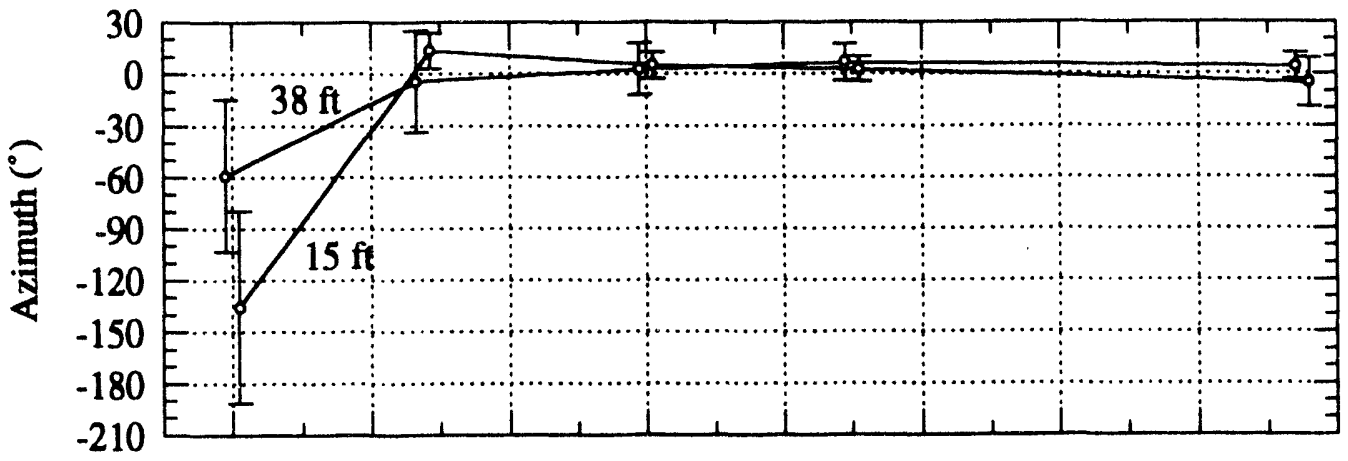

c)

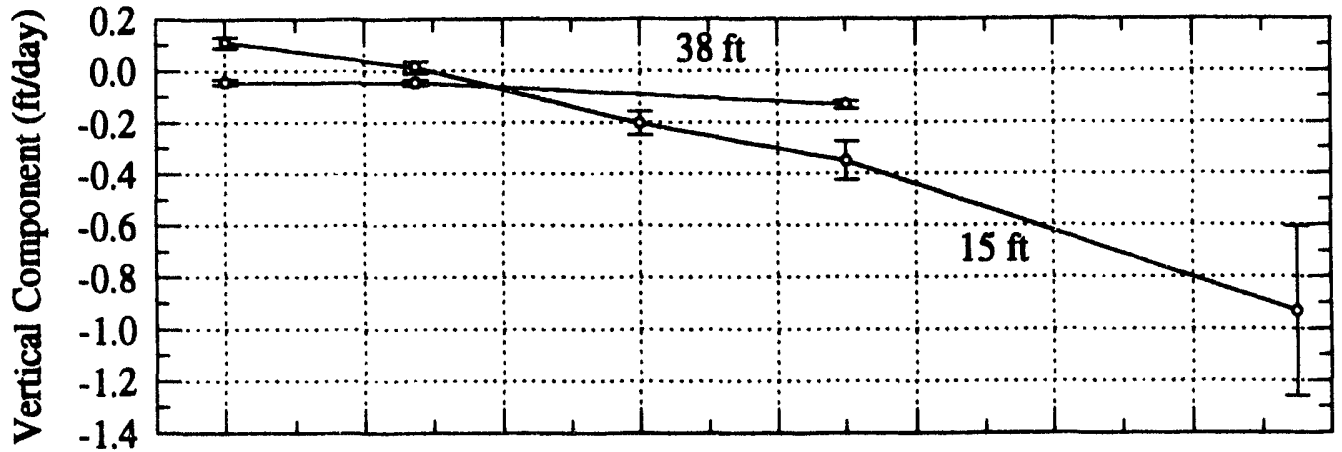

d)

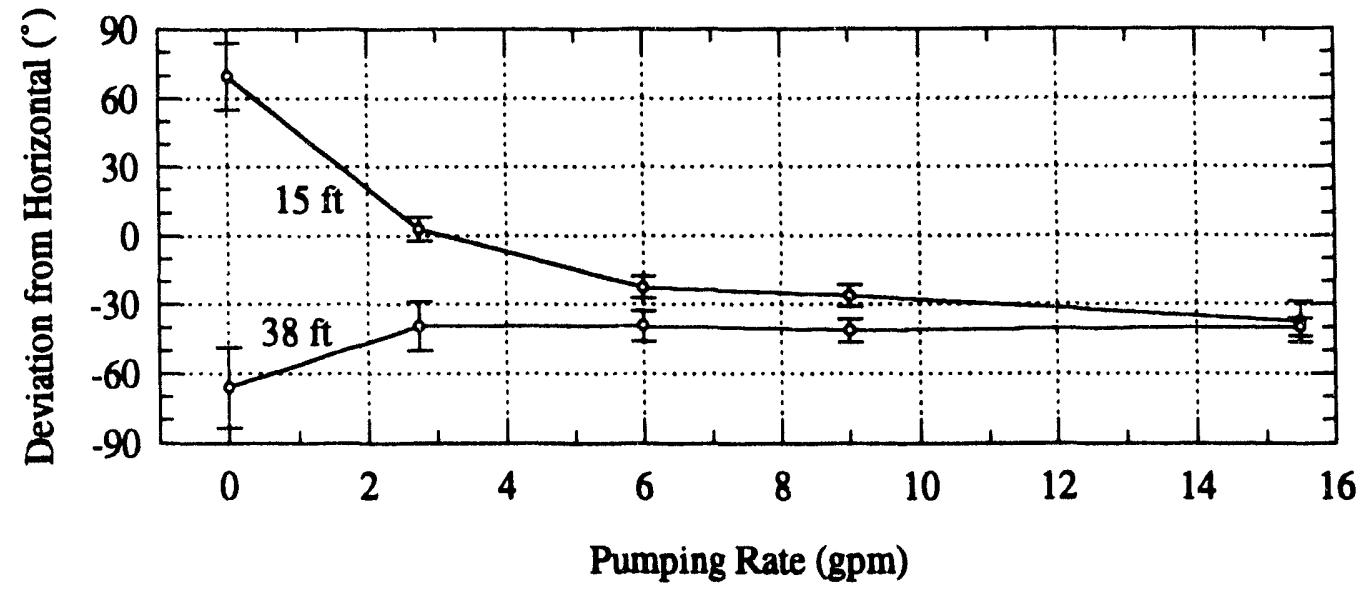

Figure 14 - Flow velocity as a function of pumping rate for the two probes. The data in a) and b) at pumping rates greater than 0 have had the horizontal component of the background flow subtracted from them. In b) the data points have been shifted slightly along the horizontal axis for clarity. 
Table 1 - Flow velocity measured at TMF1B 38 feet from the pumping well.

\begin{tabular}{|c|c|c|c|c|c|c|}
\hline $\begin{array}{c}\text { Pumping Rate } \\
(\mathrm{gpm})\end{array}$ & $\begin{array}{c}\text { Uncorrected } \\
\text { Horizontal } \\
\text { Component } \\
(\mathrm{f} / \text { day) }\end{array}$ & $\begin{array}{c}\text { Uncorrected } \\
\text { Azimuth } \\
\left({ }^{\circ}\right)\end{array}$ & $\begin{array}{c}\text { Corrected } \\
\text { Horizontal } \\
\text { Component } \\
(\mathrm{f} / \text { day) }\end{array}$ & $\begin{array}{c}\text { Corrected } \\
\text { Azimuth }\left(^{\circ}\right)\end{array}$ & $\begin{array}{c}\text { Vertical } \\
\text { Component } \\
(\mathrm{f} / \text { day) }\end{array}$ & $\begin{array}{c}\text { Deviation } \\
\text { from } \\
\text { Horizontal } \\
\left({ }^{\circ}\right)\end{array}$ \\
\hline 0.0 & $0.020 \pm 0.016$ & $-59 \pm 44$ & - & - & $-0.045 \pm 0.010$ & $-66 \pm 17$ \\
\hline 2.75 & $0.058 \pm 0.018$ & $-21 \pm 16$ & $0.044 \pm 0.024$ & $-4 \pm 29$ & $-0.048 \pm 0.011$ & $-39 \pm 11$ \\
\hline 6.0 & - & $-7 \pm 10$ & - & $3 \pm 15$ & - & $-39 \pm 7$ \\
\hline 9.0 & $0.151 \pm 0.023$ & $0 \pm 8$ & $0.141 \pm 0.028$ & $7 \pm 11$ & $-0.133 \pm 0.014$ & $-41 \pm 5$ \\
\hline 15.5 & - & $0 \pm 6$ & - & $4 \pm 8$ & - & $-40 \pm 4$ \\
\hline
\end{tabular}

Table 2 - Flow velocity measured at TMF2B 15.5 feet from the pumping well.

\begin{tabular}{|c|c|c|c|c|c|c||}
\hline $\begin{array}{c}\text { Pumping Rate } \\
(\mathrm{gpm})\end{array}$ & $\begin{array}{c}\text { Uncorrected } \\
\text { Horizontal } \\
\text { Component } \\
(\mathrm{f} / \text { day) }\end{array}$ & $\begin{array}{c}\text { Uncorrected } \\
\text { Azimuth } \\
\left({ }^{\circ}\right)\end{array}$ & $\begin{array}{c}\text { Corrected } \\
\text { Horizontal } \\
\text { Component } \\
(\mathrm{f} / \text { day })\end{array}$ & $\begin{array}{c}\text { Corrected } \\
\text { Azimuth } \\
\left({ }^{\circ}\right)\end{array}$ & $\begin{array}{c}\text { Vertical } \\
\text { Component } \\
(\mathrm{f} / \text { day) }\end{array}$ & $\begin{array}{c}\text { Deviation } \\
\text { from } \\
\text { Horizontal } \\
\left({ }^{\circ}\right)\end{array}$ \\
\hline 0.0 & $0.041 \pm 0.032$ & $-135 \pm 55$ & - & - & $0.109 \pm 0.021$ & $70 \pm 15$ \\
\hline 2.75 & $0.251 \pm 0.040$ & $8 \pm 8$ & $0.285 \pm 0.054$ & $13 \pm 10$ & $0.013 \pm 0.023$ & $3 \pm 5$ \\
\hline 6.0 & $0.497 \pm 0.075$ & $2 \pm 7$ & $0.528 \pm 0.083$ & $5 \pm 8$ & $-0.205 \pm 0.047$ & $-22 \pm 5$ \\
\hline 9.0 & $0.720 \pm 0.107$ & $1 \pm 7$ & $0.751 \pm 0.113$ & $3 \pm 7$ & $-0.352 \pm 0.076$ & $-26 \pm 5$ \\
\hline 15.5 & $1.211 \pm 0.261$ & $-7 \pm 14$ & $1.237 \pm 0.264$ & $-5 \pm 14$ & $-0.932 \pm 0.326$ & $-38 \pm 9$ \\
\hline
\end{tabular}

from the velocity measurements made after the pumping was initiated.

When the pump was operating, both probes measured significant horizontal flow velocities that increased linearly with the pumping rate. In terms of the direction of the horizontal component, the flow sensors measured velocities which were directed toward the pumping well within the estimated uncertainties of the measurements, with only one exception. At a pumping rate of $3 \mathrm{gpm}$ the flow sensor which was 15 feet from the pumping well measured a direction which was oriented $13^{\circ} \pm 10^{\circ}$ relative to the direction to the pumping well. The vertical component of the flow velocity measured by the probe in TMF1B $(R=38 \mathrm{ft})$, which started out negative, ie., downwardly directed, remained downwardly directed, and increased in magnitude only slightly. The vertical component of the flow velocity measured by the sensor which was closer to the pumping well (TMF2B, $R=15.5 \mathrm{ft}$ ), which was upwardly directed when the pump was not operating, declined to $0.01 \pm 0.02 \mathrm{ft} /$ day at $3 \mathrm{gpm}$, indicating horizontal flow, and then 
became increasingly negative with increasing pumping rate. At 3,6 and $9 \mathrm{gpm}$ the deviation of the flow velocity vector from the horizontal was significantly less at the probe located $15 \mathrm{ft}$ from the pumping well then it was at the farther probe. At $15 \mathrm{gpm}$, however, both probes measured flows which deviated from the horizontal by about $40^{\circ}$ in a downward direction.

An important consideration when using these probes is the amount of time it takes the flow sensors to respond to changes in the flow velocity around them. During the two stages of the experiment being described, the two flow sensors were essentially at equilibrium in a medium with only very low flow past them when the pumps were initially turned on at 15 and then $6 \mathrm{gpm}$. When the pump was activated, numerical modelling which will be described shortly indicates that the actual flow velocity past the probes increased very quickly and established itself at the new equilibrium value in a few minutes. As is apparent in Figures 10a, 11a, 12a and 13a, it took the flow sensors much longer to adjust to the new flow velocity. Figure 15 illustrates the approximate time required for the flow sensors to reach the new equilibrium velocity, as a function of the measured equilibrium horizontal flow velocity. The two points on the plot at 0.5 and $1.2 \mathrm{f} /$ day are derived from the flow sensor located at $15.5 \mathrm{ft}$ from the pumping well when the pumping well was being pumped at 6 and $15 \mathrm{gpm}$, respectively. The two points at 0.1 and $0.2 \mathrm{ft} / \mathrm{day}$ are derived from the flow sensor located $38 \mathrm{ft}$ from the pumping well when the pump was operating at the same rates. Since the data from the probe $38 \mathrm{ft}$ from the pumping well did not completely reach equilibrium at these pumping rates, these values are estimates obtained by extrapolating the velocity vs time data in Figures 12a and 13a to the anticipated equilibrium velocity. The data in Figure 15 indicate that the faster the velocity past the probe, the more quickly it responds to changes in the flow velocity. This is to be expected since at faster velocities heat is advected more quickly around the probe. The data in Figure 15 indicate that equilibrium is reached in the time it takes the water to travel around the probe a distance of roughly 6 inches.

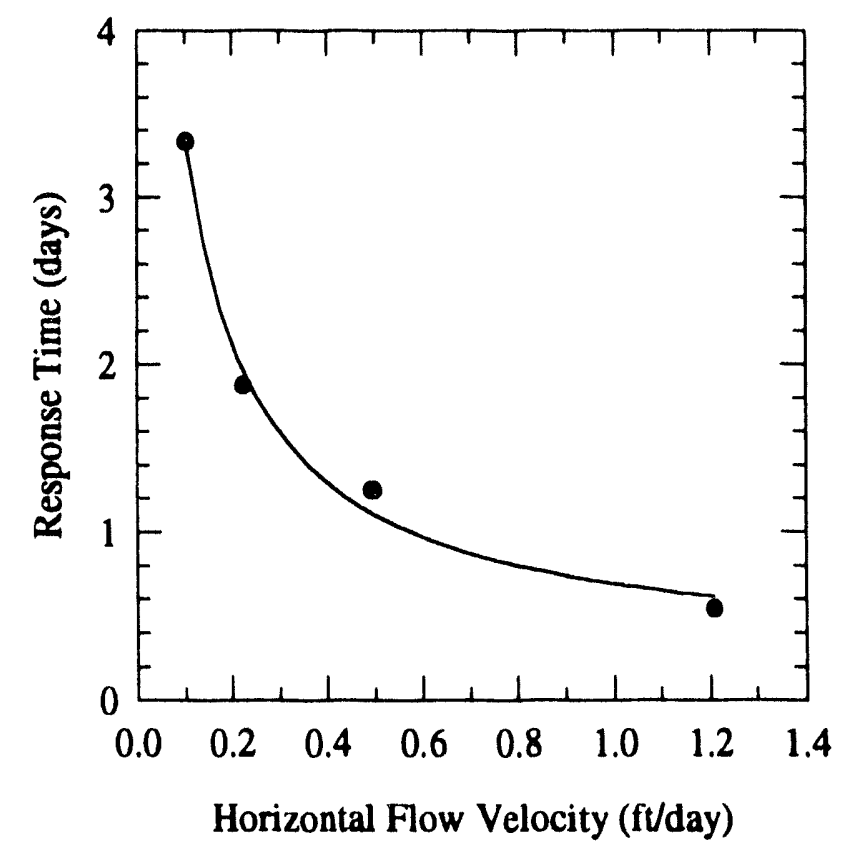

Figure 15 - Approximate time required for measured horizontal magnitude to reach equilibrium after step change in ambient flow velocity. 


\section{Analysis of Hydrologic Data}

\section{Horizontal Component}

The simplest way of estimating the true Darcy flow velocity toward the pumping well is to assume that there was no flow in the aquifer when the pump was not active, the aquitards above and below the aquifer are perfectly impermeable and that the hydrologic properties of the aquifer are perfectly homogenous and isotropic. Given these assumptions, the total amount of water per unit time flowing through the surface of a right circular cylinder whose long axis coincides with the screened interval of the pumping well is equal to the rate at which water is being pumped from the pumping well and is uniform over the surface of the cylinder (Figure 16). In this case, the direction of the Darcy velocity vector will be purely horizontal and radially directed toward the pumping well and its magnitude will equal the pumping rate divided by the surface area of the cylinder encompassing the pumping well. The magnitude can be calculated according to the formula

$$
v=\frac{Q}{2 \pi r z}
$$

where $\boldsymbol{v}$ is the magnitude of the Darcy velocity, $\boldsymbol{Q}$ is the volume of water pumped from the pumping well per unit time, $z$ is the thickness of the aquifer and $r$ is the distance from the pumping well to the observation point.

Velocities at radial distances from the pumping well corresponding to the positions of the flow sensors and at pumping rates corresponding to those used during the test, calculated according to Equation 5, are compared with the horizontal flow velocities measured with the flow sensors in Figure 17. The measured values used have had the background flow velocity, which was measured when the pump was not operating, subtracted from them. The theoretical and measured velocities from the probe in TMF1B ( $R=38 \mathrm{ft}$ ) differ by only about $11 \%$, which is less than the uncertainties in the measured values. The measured velocities from the probe in TMF2B $(R=15.5 \mathrm{ft})$ exceed the theoretical velocities by about $75 \%$; significantly more than the uncertainty in the measured values. While this correlation between the measured and theoretical velocities is considered to be quite good, it is worthwhile to seek plausible explanations for any discrepancy between them. The primary mechanism that would result in a misleading comparison between the measured and theoretical velocities is inhomogeneity of the hydraulic properties of the aquifer. There are two sources of inhomogeneity to consider: natural variations of hydraulic properties that existed in the aquifer prior to any drilling activities and variations that were introduced into the aquifer by the emplacement of the flow sensors. These will be addressed in turn.

To assess the importance of naturally

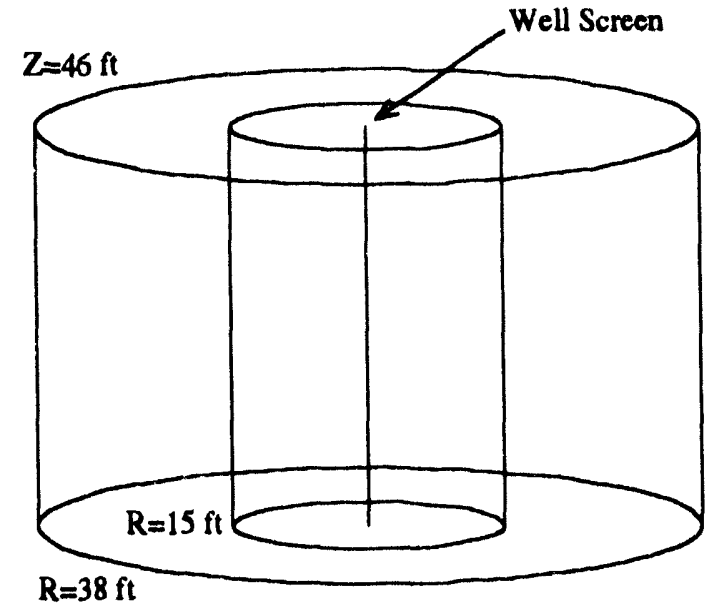

Figure 16 - Cylinders of constant flow velocity surrounding the pumping well. 
occurring inhomogeneity of the aquifer properties, a numerical model was used to calculate the drawdown expected at the piezometer wells P26M1, P26M2 and P26Al. A high degree of correlation between the calculated and measured drawdown would suggest a relatively homogeneous aquifer while significant differences between the modelled and observed drawdowns would indicate inhomogeneity and/or anisotropy in the aquifer properties. The numerical model used is the SAFT3D code developed by Hydrologic, Inc. (Huyakorn et al., 1991). This fully three dimensional finite element code simulates groundwater flow in subsurface porous media. The model used in this study consists of a 10 foot thick aquitard overlying a 46 foot thick aquifer with an impermeable bottom. The hydrologic characteristics of these two units were taken from a study of the hydrology of the site by Nichols (1993) and are listed in Table 3.

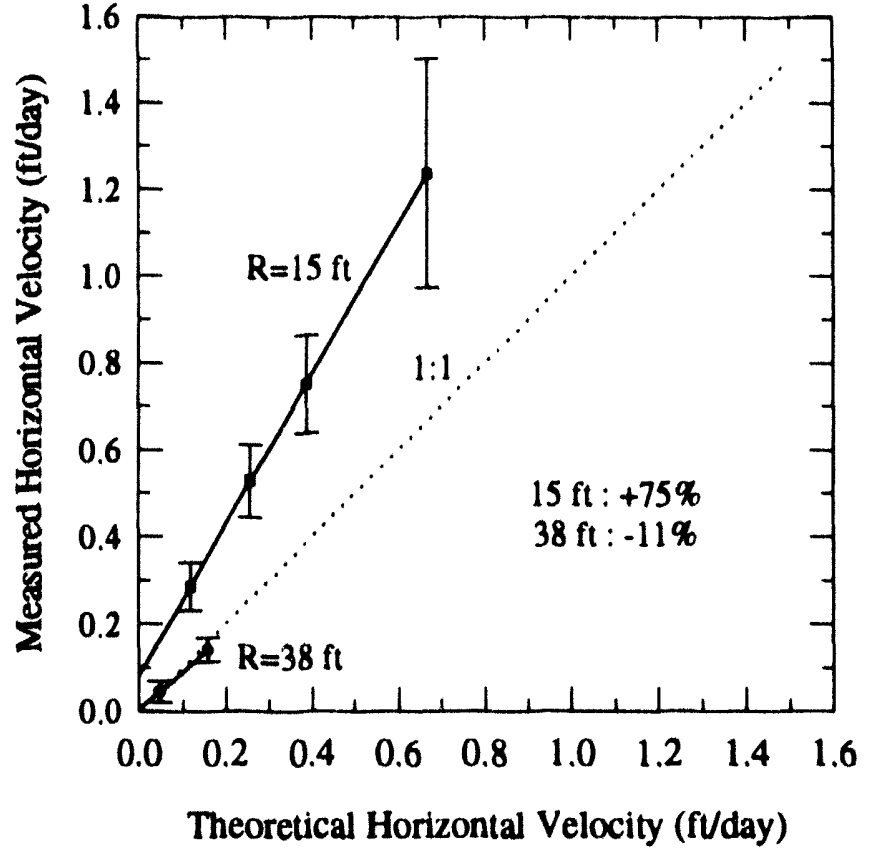

Figure 17 - Comparison of measured and theoretical horizontal flow velocities.

Table 3 - Hydrologic properties of the relevant formations at the test site.

\begin{tabular}{|c|c|c|c|c|}
\hline Unit & $\begin{array}{c}\text { Thickness } \\
(\mathrm{ft})\end{array}$ & $\begin{array}{c}\text { Effective } \\
\text { Porosity }\end{array}$ & $\begin{array}{c}\text { Hydraulic } \\
\text { Conductivity } \\
(\mathrm{ft} / \mathrm{min})\end{array}$ & $\begin{array}{c}\text { Specific } \\
\text { Storage }\end{array}$ \\
\hline Aquitard & 10 & .01 & $2.6 \times 10^{-7}$ & $5 \times 10^{-6}$ \\
\hline Aquifer & 46 & .3 & 0.01395 & $1 \times 10^{-7}$ \\
\hline
\end{tabular}

Numerically, the aquitard was modelled using one layer consisting of 60 rectangular elements in the $x$ direction and the same number in the $y$ direction. The aquifer consisted of 5 layers, each consisting of $60 \times 60$ elements. The pumping well was located at the origin in the $x-y$ plane and zero flux boundary conditions were imposed on the vertical sides of the model which were located at $+/-10,000$ feet in the $y$ direction and at -1000 and $+10,000$ feet in the $x$ direction. The three vertical boundaries at 10,000 feet distance from the pumping well were sufficiently far away that no drawdown was calculated at those edges of the model. The boundary at -1000 feet was incorporated in the model of Nichols (1993) to account for upward curvature in the observed time-drawdown curves. While the presence of this boundary affected 
the shape of the calculated time-drawdown curves, it had an insignificant effect on the radial distribution of the calculated drawdown at distances from the pumping well corresponding to the positions of the observation wells used in this study because of the close proximity of the observation wells to the pumping well. Constant head and zero flux boundary conditions were imposed at the top of the aquitard and at the bottom of the aquifer, respectively. In the model, pumping rates which correspond to those used during the pump tests were uniformly applied over the vertical extent of the aquifer. Semi-log plots of both the observed and calculated time vs drawdown data are plotted in Figure 18. The large "humps" in the observed drawdown data at 15 and $6 \mathrm{gpm}$ result from adjustments to the pumping rate made when the pump was initially turned on at the beginning of each pumping campaign. These effects do not influence the data after about 15 or 20 minutes and were not modelled numerically. To first order, the calculated and measured drawdown results match quite well.

Figure 19 illustrates the calculated and measured drawdown as a function of radial distance from the pumping well at the end of each period during which the pumping rate was held constant. Again, the measured and calculated drawdown agree quite well; differences are generally less than $0.1 \mathrm{ft}$. In detail, however, some significant differences in gradient are evident. At $15 \mathrm{gpm}$ for example, the measured and modeled drawdown agree almost exactly at a distance of $15 \mathrm{ft}$ from the pumping well but the model predicts approximately $0.1 \mathrm{ft}$ less drawdown 40 $\mathrm{ft}$ from the pumping well than was observed. These difference indicate a difference in gradient, and therefore flow velocity, between the modeled and observed situations. In Figure 20, the average modeled and observed gradients between $R=15 \mathrm{ft}$ and $R=40 \mathrm{ft}$ are compared. These gradients were calculated by dividing the difference in drawdown between $R=15$ and $R=40 \mathrm{ft}$ by the separation of the observation points $(\Delta \mathrm{h} / \Delta \mathrm{r})$. The data in Figure 20 indicate that the model predicts gradients that are about $25 \%$ higher than were actually observed. The most plausible explanation for this discrepancy is that there exists some natural heterogeneity in the horizontal hydraulic conductivity of the sediments which comprise the aquifer. Referring to the map in Figure 8a, one can speculate that the hydraulic conductivity of the aquifer material in the vicinity of the piezometer wells, P26M1, P26M2 and P26AI, is somewhat lower than the mean horizontal hydraulic conductivity of the aquifer, while that of the sediments surrounding the flow sensor in TMF2B ( $R=15 \mathrm{ft})$ is somewhat higher than average. This would explain the lower-than-expected horizontal gradient observed between the piezometer wells P26M1 and P26M2, and the higher than expected velocity observed by the flow sensor in TMF2B. These effects were not modelled numerically because there are no data describing horizontal variations in hydraulic conductivity which could be used to constrain such models.

Given that the two probes are only about 23 feet apart, naturally occurring variations in hydraulic conductivity are probably inadequate to completely explain the fact that the flow sensor in TMF1B $(R=38 \mathrm{ft})$ measured almost exactly the flow velocities predicted according to Equation 5 while the flow sensor in TMF2B ( $R=15.5 \mathrm{ft}$ ) measured flow velocities which are significantly higher than predicted. It is likely that the method used to emplace the flow sensors resulted in some enhancement of the measured horizontal component of the flow velocity over the flow velocity one would observe if the flow sensor were not there. As described previously, the holes in which the flow sensors were emplaced were drilled with a 6 inch diameter hollow stem auger. The aquifer material which was replaced by the borehole was removed from the subsurface during the drilling process. After the probes were lowered down the center of the auger, the auger was retracted and the soil collapsed around the 2 inch diameter probes. This process created a disturbed zone around the probe which extends radially out from the probe some 

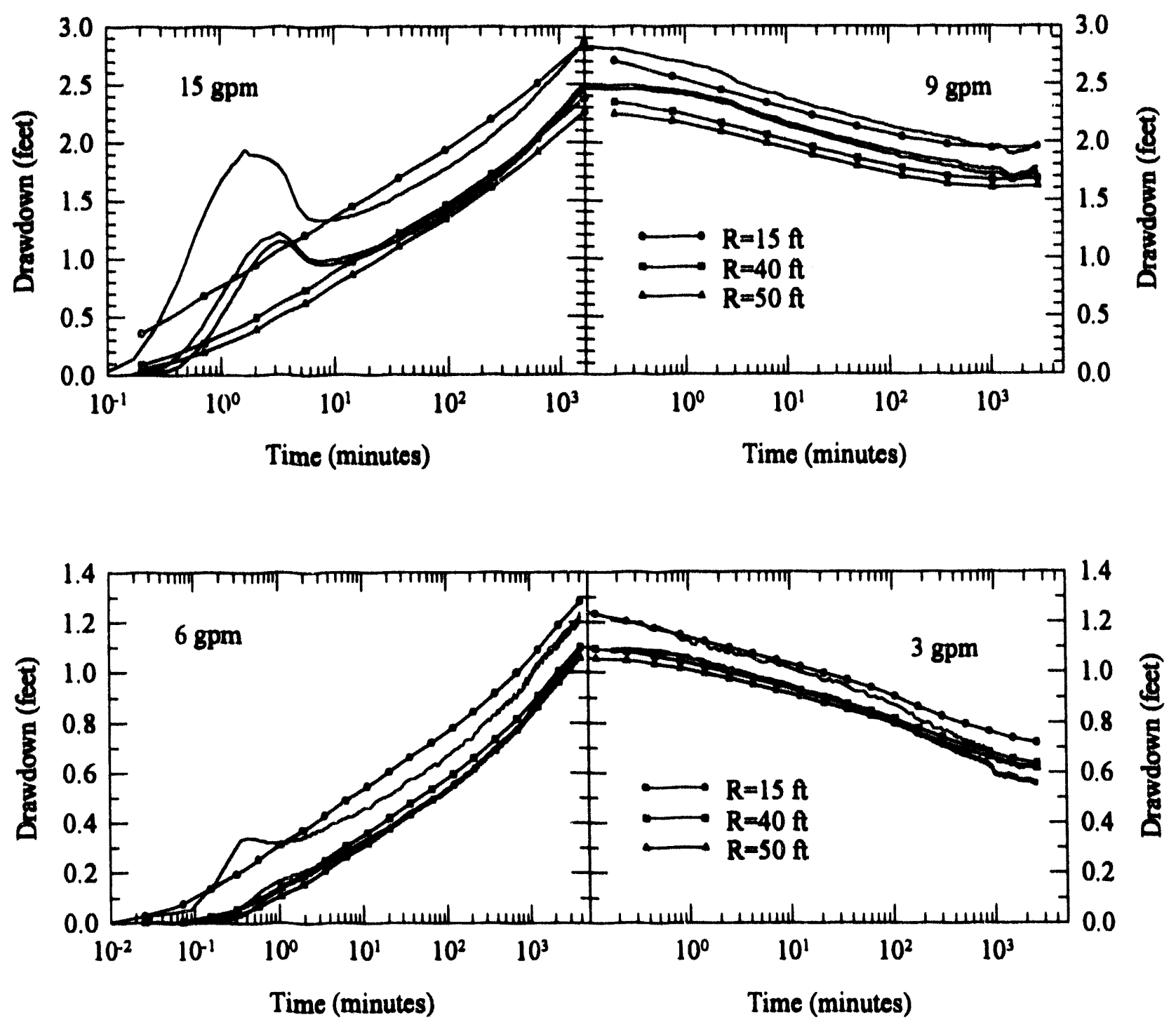

Figure 18 - Drawdown vs. log time at different distances from the pumping well. Symbols represent the results of the numerical model while the curves are observed values. 


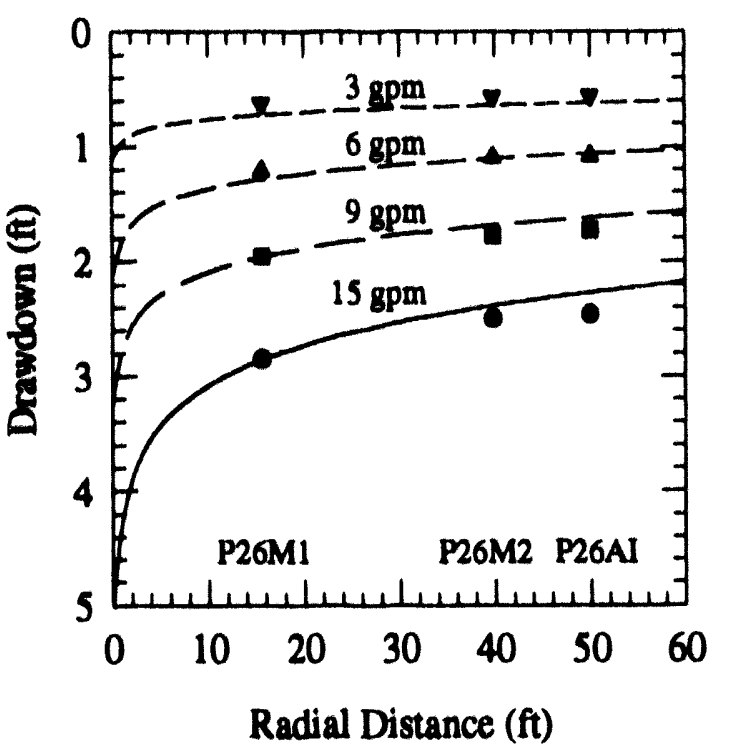

Figure 19 - Drawdown vs radial distance from the pumping well for several different pumping rates. Symbols represent observed values, curves are the results of numerical modelling.

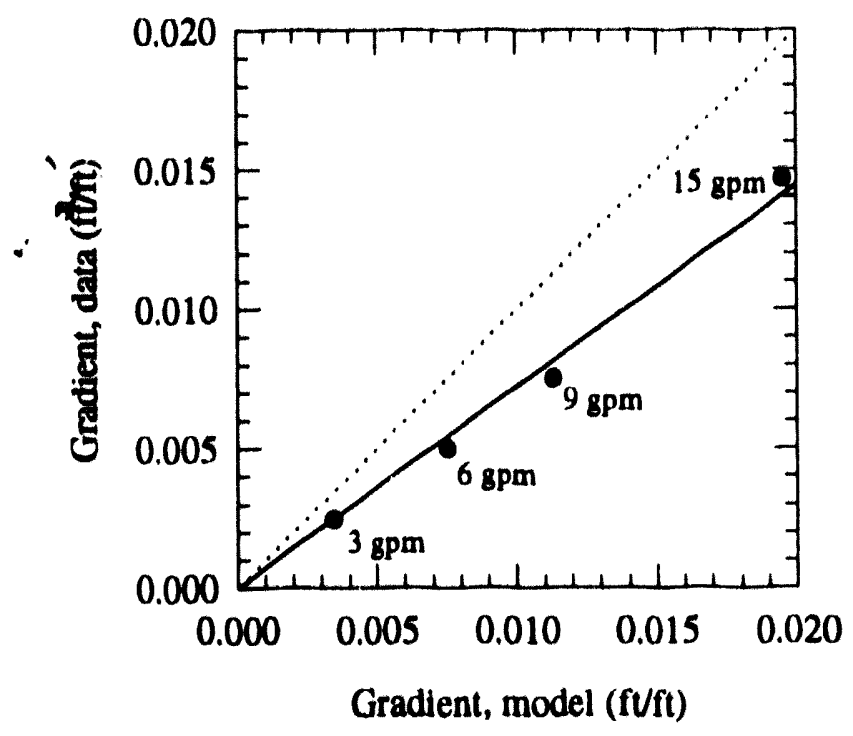

Figure 20 - Comparison of observed and modeled hydraulic gradients at different pumping rates.

distance, probably on the order of one to two feet. Since material in the formation expanded to fill a void, this disturbed zone would have higher porosity and hydraulic conductivity than the surrounding material. As a result, horizontal flow past the probe would be enhanced in the immediate vicinity of the probe as the horizontal flow lines in the aquifer converge within the zone of increased permeability around the probe (Wheatcraft and Winterberg, 1985). Since the soil probably collapsed around the probes in some highly irregular and complex manner, the degree and distribution of the soil disturbance, and the amount by which the flow around the probes was enhanced, cannot be calculated or corrected for. It is possible that the amount of disturbance created during the emplacement of the probe in TMF2B was greater than that created during deployment of the probe in TMF1B, which would explain the fact that the horizontal velocities measured by the former differed significantly from the predicted velocity while those measured by the latter did not.

\section{Vertical Component}

If perfect homogeneity of the aquifer material is assumed then the groundwater flow in the aquifer surrounding the pumping well should be purely horizontal and radially directed toward the pumping well. In fact, a significant vertical component to the flow was observed by the flow sensors. A possible explanation for these observations is the existence of significant variations in the horizontal component of the hydraulic conductivity of the aquifer material as a function of depth in the aquifer. If this were the case then groundwater in relatively low conductivity layers would tend to flow vertically toward levels in the aquifer with higher horizontal 
conductivity, imparting a significant vertical component to the flow velocity. There are some data to suggest that such vertical variations in the horizontal hydraulic conductivity exist within the aquifer being considered. The hydraulic conductivity of aquifer sediment samples obtained from wells which penetrate the aquifer in the general vicinity of the test site were estimated using grain size analysis. While hydraulic conductivity values determined from grain size analysis are generally considered to be unreliable (Taylor, et al., 1990) they can be used to constrain the relative vertical distribution of hydraulic conductivity in the aquifer. The grain size analysis results were used to determine what effects vertical variations in hydraulic conductivity might have on the flow field surrounding the pumping well.

The first problem with the results of the grain size analysis is that the mean hydraulic conductivity of the aquifer determined from the grain size analysis is larger than the value determined from the pump tests by a factor of approximately 8 . To correct for this, the grain size analysis results were reduced by this factor to bring them into agreement with the pump test results. Then the aquifer was divided into 7 layers of different hydraulic conductivity for purposes of numerical modeling. Both the corrected hydraulic conductivity data from the grain size analysis and the conductivity of the seven layers used in the numerical model are illustrated in Figure 21. The general pattern of the hydraulic conductivity suggests that the upper part of the aquifer has approximately the average hydraulic conductivity of the aquifer, there is a low conductivity horizon in the middle part of the aquifer and a relatively high conductivity layer in the bottom part of the aquifer. The flow sensors were located about $22.5 \mathrm{ft}$ above the base of the aquifer, roughly in between the low and high conductivity layers. In this position, a significant downwardly directed vertical component to the flow is expected.

Figure 22a illustrates the modeled downward component of the flow velocity as a function of vertical position in the aquifer for a pumping rate of $15 \mathrm{gpm}$. At the top of the aquifer, there is a small, downwardly directed component to the flow velocity. The magnitude of the downwardly directed flow increases in the low conductivity layer near the middle of the aquifer and then reaches a maximum value in the transition layers between the low and high conductivity layers. In the bottom-most layer, the flow velocity has a small upwardly directed component, toward the high conductivity layer directly above it. The magnitude of the downwardly directed flow is larger at a radial distance of $15 \mathrm{ft}$ than it is at 38

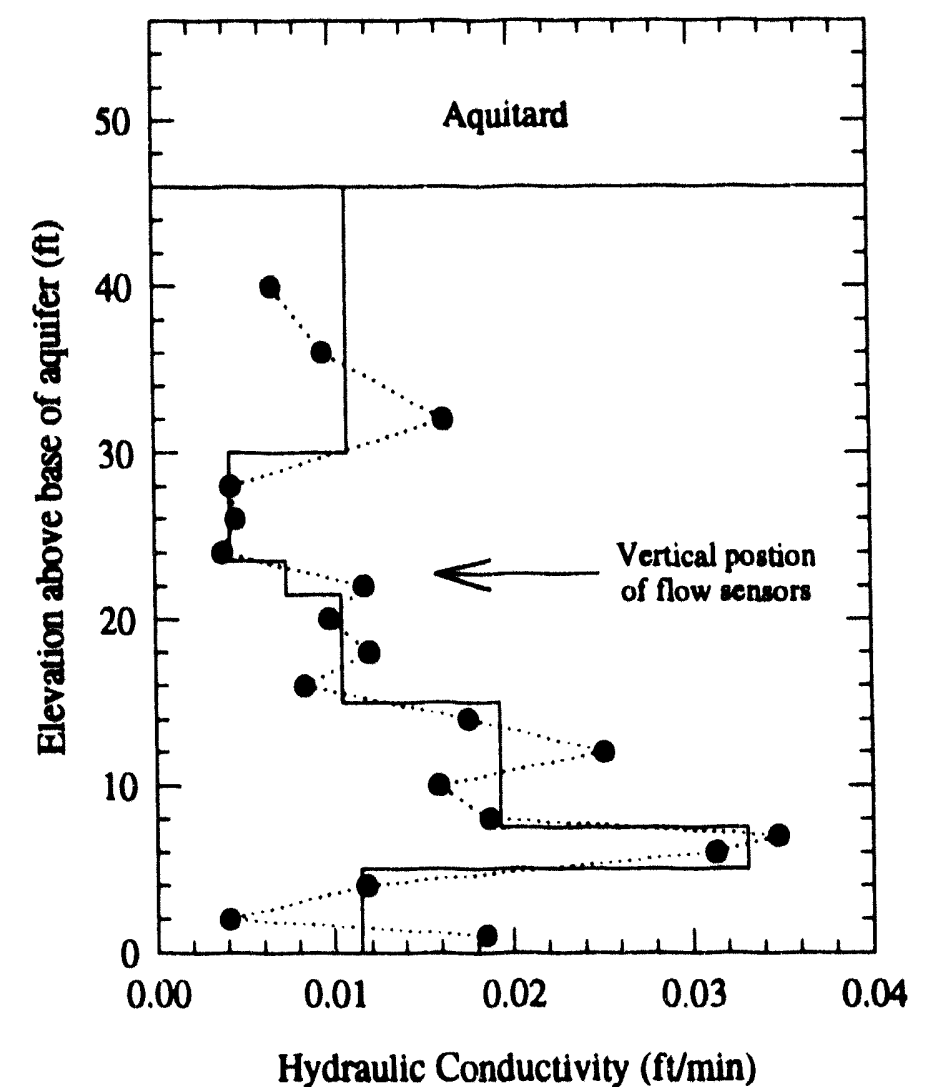

Figure 21 - Vertical distribution of hydraulic conductivity in the aquifer. Symbols represent the grain size analysis data, adjusted to a mean value of $0.014 \mathrm{f} / \mathrm{min}$. The solid line represents values used in the numerical model. 
feet. Figure 22b illustrates the angle between the flow velocity vector and the horizontal plane, with downward angles positive. The vertical position of the maximum downward deviation from the horizontal is at a slightly higher position within the aquifer than is the maximum vertical flow velocity. This maximum downward deviation of the flow velocity occurs at the vertical position of the flow sensors. The maximum deviations from the horizontal predicted by the model are only about $4^{\circ}$ and $8^{\circ}$ at distances of $15 \mathrm{ft}$ and $38 \mathrm{ft}$, respectively. While considerably less than the downward deviations of approximately $30^{\circ}$ to $40^{\circ}$ observed by the flow sensors, the modeling results indicate, at least qualitatively, that a downwardly directed component of the flow velocity is expected at the position of the flow sensors. Also note that the downward deviation from the horizontal is larger at greater radial distance from the pumping well, indicating the dominance of horizontal flow in close proximity to the pumping well. While this pattern is observed in the flow sensor data at the lower pumping rates (Figure 14d), the measured downward deviation from the horizontal is about the same at $R=15.5 \mathrm{ft}$ and $R=38 \mathrm{ft}$ when the pumping rate was $15 \mathrm{gpm}$.

A plausible reason for the larger than expected observed vertical components is that a

a)

$$
\text { Hydraulic Conductivity Model (f } \mathrm{f} / \mathrm{min} \text { ) }
$$

b) Hydraulic Conductivity Model (f $/ \mathrm{min}$ )
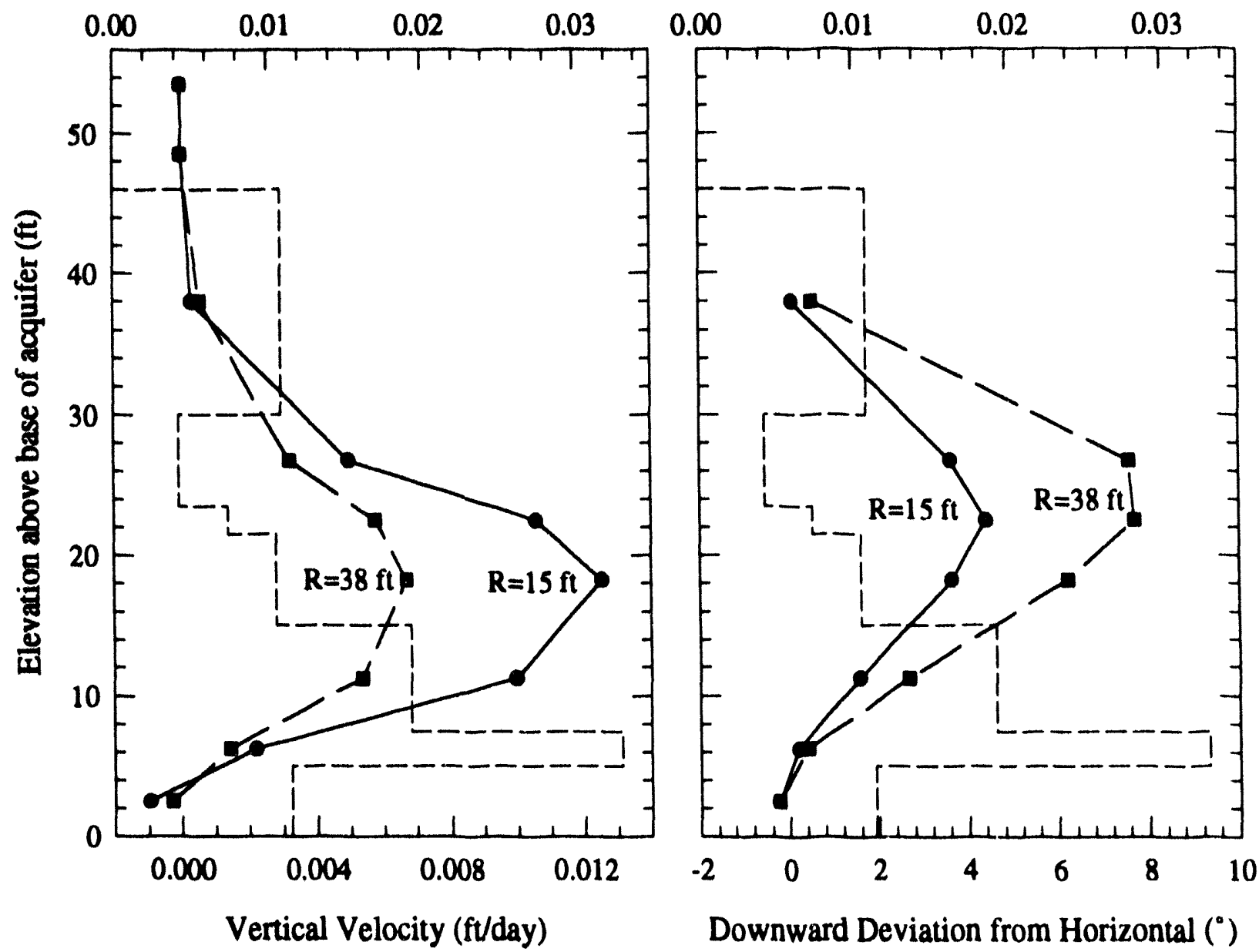

Figure 22 - Symbols represent a) the vertical component of the flow velocity and b) the downward deviation from the horizontal vs depth. Dashed lines in both plots are the hydraulic conductivity model. 
relatively high permeability pathway was created around the flow sensors when they were installed. The sensor emplacement process used probably introduced vertical conduits of relatively high hydraulic conductivity through the aquifer. These conduits probably do not extend into the unconfined aquifer above the upper aquitard since the holes were sealed with bentonite seals in the upper clay unit. The conduits extend from the top of the aquifer down to a depth of about $5 \mathrm{ft}$ below the probes. The conduits extend below the bottom of the probes because the holes were drilled to that depth to circumvent emplacement difficulties which resulted from flowing sand running up inside the augers. Since these conduits connect the low hydraulic conductivity layer above the probes with the higher conductivity layers below the probes it is to be expected that the naturally occurring downward component of flow would be significantly enhanced in the vicinity of the flow sensors as compared to the undisturbed formation. No attempt has been made to model this effect because the geometry and hydraulic properties of the conduit created by the drilling are completely unknown.

\section{Discussion}

The goal of the test described in this report is to ascertain the usefulness of the In Situ Permeable Flow Sensors for measuring groundwater flow velocity. Two relevant questions are whether or not the flow sensors are responsive to changes in flow velocity and, secondly, do the measured values accurately reflect the true velocities. The linearity of the relationship between the pumping rate and the measured magnitude of the horizontal component of the flow velocity (Figure 14a) and the excellent correspondence between the measured orientation of the horizontal component of the flow velocity vector and the direction toward the pumping well (Figure 14b) are highly encouraging in that they suggest that the flow sensors are in fact responsive to the groundwater flow velocity.

The question of whether or not the velocities measured with the flow sensors accurately represent the true flow velocities is more difficult to answer. As far as this test is concerned, there are four representations of the flow velocity field during the test: the velocity measurements obtained with the flow sensors, quantitative models of the flow velocity field, conceptual models of the velocity field and the true velocity. The true flow velocity field includes all the complexities which result from both naturally occurring and artificially introduced spatial variations in hydraulic conductivity; it is what one would measure with an ideal flowmeter. What is desired is a direct comparison of the flow sensor measurements with the truth. This is of course impossible since the true flow velocity cannot be known. The approach adopted here is to first construct quantitative models which either incorporate, or are constrained by, available data. Next, conceptual models are envisioned which incorporate plausible hydrologic features which are neither prescribed nor precluded by any available data. These conceptual models are used to qualitatively address any remaining discrepancies between the measured velocities and those predicted by the quantitative models. Finally, it is hoped that the conceptual models bear some resemblance to reality.

The first and simplest quantitative model, described by Equation 5 , is based on the assumption that the hydrologic properties of the aquifer are perfectly homogeneous. The measured and predicted horizontal flow velocities compare quite well. The magnitudes of the horizontal flow velocities measured by the two flow sensors differ from the model velocities by only $-11 \%$ and $75 \%$. Despite this reasonably good agreement between the measured and modelled horizontal flow velocities, there remain some differences which warrant further 
investigation. The first is that one of the flow sensors measured horizontal components of flow which were consistent with the model, within the uncertainty of the measurements, while the other flow sensor observed significantly larger horizontal velocities than the model would predict. The second discrepancy between the measured and modelled flow velocities is that significant vertical components of flow were measured by both flow sensors while the simple model described by Equation 5 predicts purely horizontal flow.

To address the first issue, a numerical model was developed which differed only slightly from the analytical model described by Equation 5 . The numerical model still assumed perfect homogeneity of the hydraulic properties of the aquifer. This model was used to calculate the hydraulic head at the location of the piezometer wells. The hydraulic gradients derived from the observations in the piezometer wells were about $25 \%$ lower than those predicted by the model, suggesting that the assumption that the horizontal distribution of hydraulic conductivity was homogeneous, is not valid. A conceptual model in which the hydraulic conductivity of the aquifer in the vicinity of the piezometer wells is lower than the average conductivity of the aquifer and the conductivity in the area around the probes is higher than average, is consistent with the hydraulic head observations and the velocity measurements in TMF2B $(R=15 \mathrm{ft})$.

Because the two flow sensors are only about 23 feet apart, naturally occurring spatial variations in hydraulic conductivity are likely to be insufficient to explain the fact that one flow sensor agreed so well with the predicted horizontal velocity while the other did not. A possible explanation for this observation is that a zone of enhanced hydraulic conductivity extending radially out from the probe a distance of one to two feet was created during the emplacement of the flow sensors and that the effect was more pronounced for the probe in TMF2B $(R=15 \mathrm{ft})$ than it was for the probe in TMF1B $(R=38 \mathrm{ft})$.

To account for the substantial vertical flow velocities observed with the flow sensors, a numerical model incorporating horizontal layers of different hydraulic conductivity was developed. This model was constrained by hydraulic conductivity values determined by grain size analysis of core samples from a well near the test site. Like the measurements, the model predicted significant downwardly directed vertical components of flow. The measured vertical components are significantly larger than those predicted by the model, however. A likely explanation for the larger than expected measured vertical flow velocities is that a vertical conduit of high hydraulic conductivity material was created within the aquifer during emplacement of the flow sensors. These conduits, which are probably on the order of a few feet in diameter, extend from the top of the aquifer down to a depth about 5 feet below the position of the flow sensors. Since these conduits connect the relatively low hydraulic conductivity layers above the probes with higher conductivity layers below the probes, significantly enhanced downward flow would be expected within them.

The analysis presented suggests that disturbance of the hydraulic properties of the formation created during emplacement of the flow sensors might significantly alter the flow velocity that is to be measured. Alternative techniques for sensor emplacement that would reduce the amount of disturbance need to be considered. A method that would likely be greatly superior to the technique used in this study would be to push the flow sensors directly into the ground with a cone penetrometer. This technique would compact the sediments around the flow sensors somewhat since no material is actually removed from the subsurface. However, the compaction would probably only extend a few inches, at most, away from the probe in a radial direction. Other actions which would reduce the disturbance to the natural flow field would be to drill no deeper than absolutely necessary to emplace the sensor and to grout the hole above the location 
of the flow sensor to minimize flow along the vertical conduit created during emplacement of the sensor.

\section{Conclusion}

Field tests of In Situ Permeable Flow Sensors in a confined aquifer at the Savannah River Site in South Carolina suggest that the technology is capable of measuring quite low levels of groundwater flow velocity in saturated geologic materials. As expected, the horizontal components of groundwater flow measured by the flow sensors pointed toward the pumping well within the uncertainties of the measurements $\left( \pm 7^{\circ}\right.$ to $\pm 30^{\circ}$, depending on pumping rate). The magnitude of the horizontal component of the flow velocity increased in direct proportion to the pumping rate, as predicted by theoretical considerations. If it is assumed that the hydraulic properties of the aquifer are homogenous and isotropic, then the magnitudes of the horizontal components of the flow velocity measured by the flow sensors agree with the expected horizontal velocities within a factor of two. Discrepancies can be explained, largely on a qualitative basis, as being the result of naturally occurring and artificially introduced spatial variations in hydraulic conductivity. From this it is possible to conclude that the flow sensors probably did a reasonably good job of measuring the true flow velocity during the test. As more experience is gained with this new technology and more confidence in the results obtained with it is acquired, it will be possible to use the technology to characterize aquifer heterogeneity rather than trying to avoid it.

It is very likely that emplacing the flow sensors with a cone penetrometer technique would greatly reduce the disturbance to the hydraulic properties of the formation compared to the emplacement technique used in this study. This would in turn reduce the uncertainty in the measured flow velocities caused by these disturbances.

Based on the encouraging results obtained with the flow sensors in this study it is hoped that the technology will find widespread applications in the areas of environmental characterization and remediation monitoring. Currently on-going activities include the use of the technology to monitor an air-stripping/bioremediation experiment at the Savannah River Site in South Carolina and a study of the interaction between groundwater and Columbia River water at the Hanford Site in Washington State. Commercialization of the technology is also underway.

\section{References}

Ballard, S., 1992a, An In Situ Permeable Flow Sensor to Monitor Groundwater Flow, Sensors Magazine, v. 9, n. 13, p. 20-26.

Ballard, S., 1992b, In Situ Permeable Flow Sensors at the Savannah River Integrated Demonstration: Phase I Results, SAND92-1952, Sandia National Laboratories, Albuquerque, New Mexico.

Caceci, M. S. and W. P. Cacheris, 1984, Fitting curves to data, Byte, v. 9, no. 5, p. 340-362.

Huyakom, P. S., S. Panday and T. Birdie, 1991, SAFT3D subsurface analysis finite element model for flow and transport in 3 dimensions, version 1.3, documentation and user's guide, Hydrogeologic, Inc., Herndon, Virginia. 
Kearl, P. M., E. G. Gardner and M. J. Gunderson, 1993, Groundwater flow delineation study at the Massachusetts Military Reserve using the Colloidal Borescope, ORNL/TM-12139, Oak Ridge, $\mathrm{TN}$.

Kearl, P. M. and C. M. Case, 1992, Direct field measurement of groundwater velocity, Interdisciplinary approaches to hydrology and hydrogeology, American Institute of Hydrology.

Kerfoot, W. B., 1988, Monitoring well construction and recommended procedures for direct ground-water flow measurements using a heat-pulsing flowmeter, Ground-water contamination: field methods, ASTM STP 963. A. G. Collins and A. I. Johnson, Eds., American Society for Testing and Materials, Philadelphia, p. 146-161.

Kerfoot, W. B. and V. A. Massard, 1985, Monitoring well screen influence on direct flowmeter measurements, Groundwater Monitoring Review, v. 5, n. 4, p. 74-77.

Melville, J. G., F. J. Molz and O. Güven, 1985, Laboratory investigation and analysis of a ground-water flowmeter, Groundwater, v. 23, n. 4, p. 486-495.

Nichols, R. L., 1993, Characterization of Shallow Groundwater at TNX, Westinghouse Savannah River Company Internal Report, WSRC-TR-92-508, 50 p.

Press, H. W., B. P. Flannery, S. A. Teukolsky, W. T. Vetterling, 1986, Numerical Recipes, The Art of Scientific Computing, Chapter 14, Section 5, Cambridge University Press.

Romero, L. A., in press, Forced convection past a slender body in a saturated porous medium, submitted to Journal of Applied Mathematics.

Taylor, K., S. Wheatcraft, J. Hess, J. Hayworth and F. Molz, 1990, Evaluation of methods for determining the vertical distribution of hydraulic conductivity, Groundwater, v. 28, n. 1, p. 88-98.

Wheatcraft, S. W. and F. Winterberg, 1985, Steady state flow passing through a cylinder of permeability different from the surrounding medium, Water Resources Research, v. 21, n. 12 , p. $1923-1929$. 


\section{Distribution}

1 Daniel B. Anderson

Pacific Northwest Laboratory

P. O. Box 999, MS P7-41

Richland, WA 99352

1 John Barich

Environmental Protection Agency

1200 Sixth Avenue,

Mail Stop, ES-098

Seattle, WA 98101

1 Theresa M. Bergsman

Pacific Northwest Laboratory

P. O. Box 999, MS P7-41

Richland, WA 99352

1 Tom Brouns

Battelle NW Labs

P. O. Box 999

Battelle Blvd.

P7-41

Richland, WA 99352

1 Charles Carrigan

Earth Sciences Division, L-206

Lawrence Livermore National Lab

P. O. Box 808

Livermore, CA 94550

1 Paul Daley

Lawrence Livermore National Lab

P. O. Box 808

Livermore, CA 94550

1 Gaynor W. Dawson

EG\&G Environmental

Route 1, Box 5038

Richland, WA 99352

5 Carol Eddy

Environmental Sciences Section

Bldg. 773-42A

Savannah River Technology Center

Aiken, SC 29808
1 John Evans

Battelle NE Lab - Sigma 5 K6-81

P. O. Box 999

Richland, WA 99352

1 K. D. Gerdes

DOE Office of Environmental

Restoration and Waste Management

Trevion II Building

Germantown, MD 20874

100 Jim Gibson

SIE, Inc.

7450 Winscott Rd,

Fort Worth, TX 76126

1 Tyler J. Gilmore

Pacific Northwest Laboratory

P. O. Box 999, MS K6-84

Richland, WA 99352

1 Steve Gorelick

Stanford University

Department of Geological and

Environmental Sciences

Stanford, CA 94305-2115

1 Bill Haas

Ames Laboratory

7 Spedding Hall

Iowa State University

Ames, IA 50011

1 Michael C. Hagood

Westinghouse Hanford Company

P. O. Box 1970, MS H6-04

Richland, WA 99352

1 Harry Hardee

Department of Mechanical

Engineering

P. O. Box $30001 /$ Dept. 3450

Las Cruces, NM 88003-00011 
1 Bob Henckel

P. O. Box 1970

MSIN H4-55

Richland, WA 99352

1 Kent M. Hodgson

Westinghouse Hanford Company

P. O. Box 1970, MS S4-25

Richland, WA 99352

1 Alan Jackman

Dept. of Chemical Engineering

University of California

Davis, CA 95616-5294

1 George J. Jackson

Westinghouse Hanford Company

P. O. Box 1970, MS H6-04

Richland, WA 99352

1 Roger Jenkins

MS 6120, Bldg. 4500 South

Oak Ridge National Laboratory

P. O. Box 2008

Oak Ridge, TN 37831-6120

1 Greg Junk

Ames Lab

7 Spedding Hall

Iowa State University

Ames, IA 50011

1 Dawn Kaback

Colorado Center for Environmental Management

Suite 2750

999 18th St.

Denver, CO 80202

1 Pete Kearl

Environmental Science Division

Oak Ridge National Laboratory

P. O. Box 2567

Grand Junction, CO 81502
1 Kim J. Koegler

Westinghouse Hanford Company

P. O. Box 1970, MS H6-05

Richland, WA 99352

1 Eric Koglin

USEPA EINSL-LV QAD

P. O. Box 93478

Las Vegas, NV 89193-3478

1 Kevin Langry

University of California

Lawrence Livermore National Laboratory

Environmental Sciences Division

P. O. Box 5507, L-524

Livermore, CA 94550

1 George V. Last

Pacific Northwest Laboratory

P. O. Box 999, MS K6-96

Richland, WA 99352

1 Robert J. Lenhard

Pacific Northwest Laboratory

P. O. Box 999, MS K6-77

Richland, WA 99352

1 Richard E. Lewis

Pacific Northwest Laboratory

P. O. Box 999, MS K6-84

Richland, WA 99352

1 Brian Looney

Environmental Sciences Section

Bldg. 773-42A

Savannah River Technology Center

Aiken, SC 29808

1 Stuart P. Luttrell

Pacific Northwest Laboratory

P. O. Box 999, MS K6-96

Richland, WA 99352 
1 Lance S. Mamiya

US Department of Energy

Richland Field Office

P. O. Box 550, MS K8-50

Richland, WA 99352

1 Gregory W. McLellan

Westinghouse Hanford Company

P. O. Box 1970, MS N3-05

Richland, WA 99352

1 Donald J. Moak

Westinghouse Hanford Company

P. O. Box 1970, MS N3-05

Richland, WA 99352

1 Darrell R. Newcomer

Pacific Northwest Laboratory

P. O. Box 999, MS K6-96

Richland, WA 99352

3 Ralph Nichols

Environmental Sciences Section

Bldg. 773-42A

Savannah River Technology Center

Aiken, SC 29808

1 Bob Peterson

4601 Mallard Court

West Richland, WA 99352

1 Abe Ramirez

Lawrence Livermore National Lab

P. O. Box 808

L-206

Livermore, CA 94550

1 Virginia Rohay

MSIN H6-06

Westinghouse Hanford Co.

P. O. Box 1970

Richland, WA 99352
1 Nina Rosenburg

Mail Stop F665

Los Alamos National Lab

Los Alamos, NM 87545

1 Joe Rossabe

Environmental Sciences Section

Bldg. 773-42A

Savannah River Technology Center

Aiken, SC 29808

1 Douglas R. Sherwood

Environmental Protection Agency

712 Swift, Mail Stop B5-01

Richland, WA 99352

1 Rodney S. Skeen

Pacific Northwest Laboratory

P. O. Box 999, MS P7-41

Richland, WA 99352

1 Steven L. Stein

Pacific Northwest Laboratory

Seattle Research Center

4000 NE 41st Street

Seattle, WA 98105

1 Deborah E. Trader

US Department of Energy

Richland Field Office

P. O. Box 550, MS K8-50

Richland, WA 99352

1 Stanley D. Tomich

Pacific Northwest Laboratory

P. O. Box 999, MS K6-08

Richland, WA 99352

1 Steve J. Trent

Westinghouse Hanford Company

P. O. Box 1970, MS H6-06

Richland, WA 99352 
1 Michael J. Truex

Pacific Northwest Laboratory

P. O. Box 999, MS P7-41

Richland, WA 99352

1 Doug Vaught

Westinghouse Hanford Co.

PO Box 1970

MS H6-05

Richland, WA 99352

1 Terry Walton

Batelle PNL

P. O. Box 999

MS K7-90

Richland, WA 99352

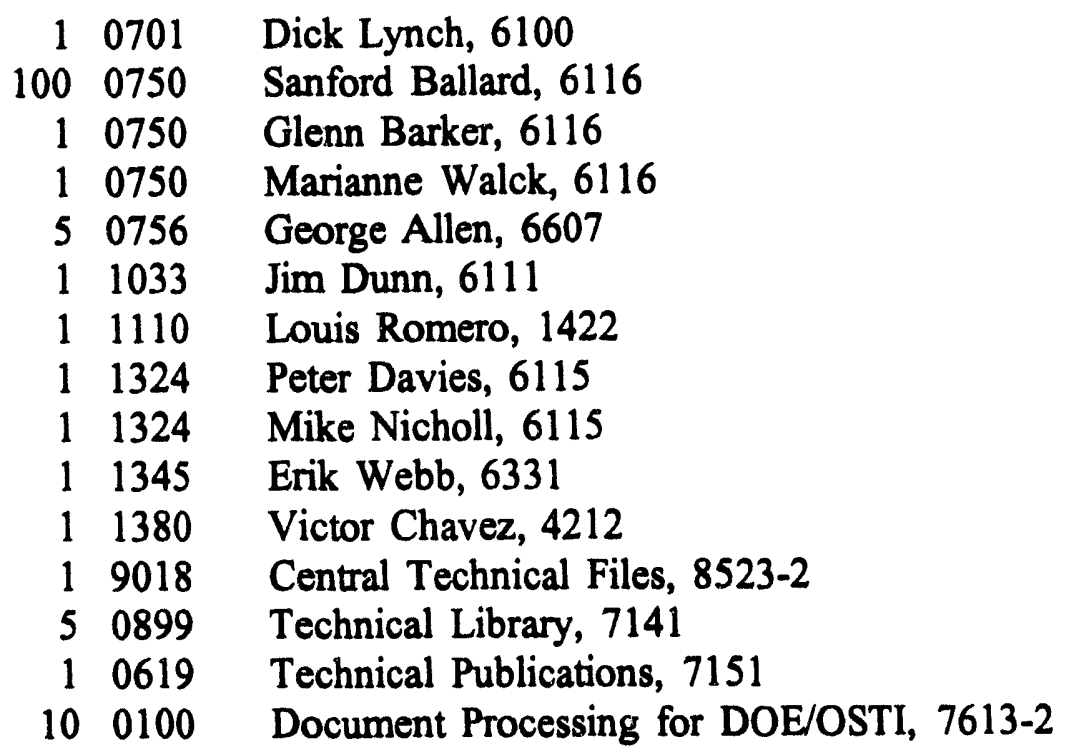



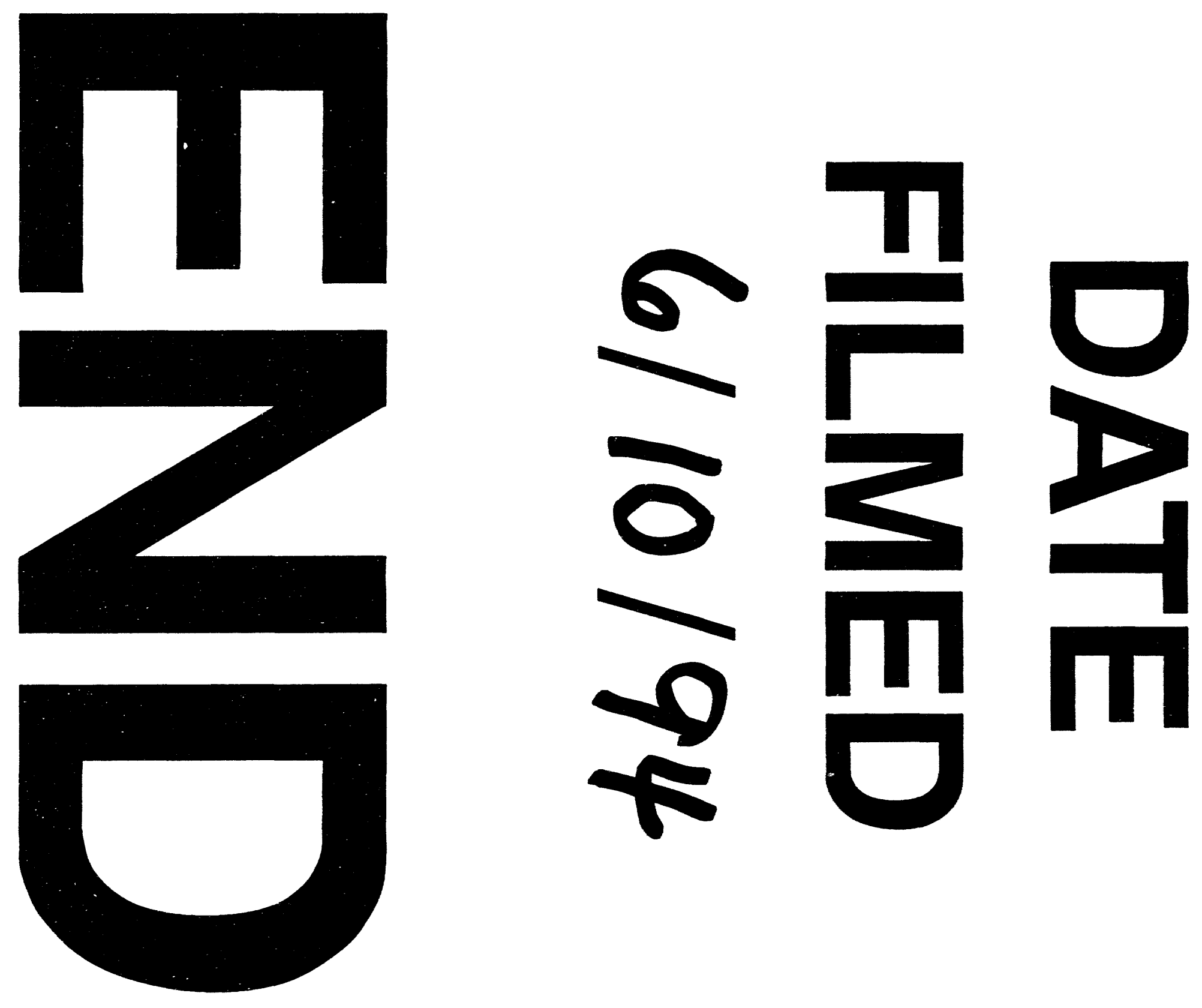
